\title{
Rapid reconstruction of SARS-CoV-2 using a synthetic genomics platform
}

https://doi.org/10.1038/s41586-020-2294-9

Received: 20 February 2020

Accepted: 24 April 2020

Published online: 4 May 2020

Check for updates

\author{
Tran Thi Nhu Thao ${ }^{1,2,3,10}$, Fabien Labroussaa ${ }^{2,4,10}$, Nadine Ebert ${ }^{1,2,10}$, Philip V'kovski ${ }^{1,2}$, \\ Hanspeter Stalder ${ }^{1,2}$, Jasmine Portmann ${ }^{1,2}$, Jenna Kelly,2, Silvio Steiner ${ }^{1,2,3}$, \\ Melle Holwerda ${ }^{1,2,3,5}$, Annika Kratzel ${ }^{1,2,3}$, Mitra Gultom ${ }^{1,2,3,5}$, Kimberly Schmied ${ }^{1,2}$,

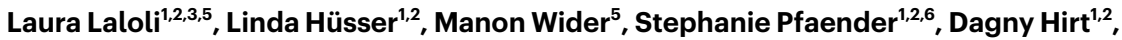 \\ Valentina Cippà ${ }^{2,4}$, Silvia Crespo-Pomar ${ }^{2,4}$, Simon Schröder ${ }^{7}$, Doreen Muth ${ }^{7,8}$, \\ Daniela Niemeyer ${ }^{7,8}$, Victor M. Corman ${ }^{7,8}$, Marcel A. Müller ${ }^{7,8,9}$, Christian Drosten $^{7,8}$, \\ Ronald Dijkman $^{1,2,5}$, Joerg Jores ${ }^{2,4,11 \bowtie} \&$ Volker Thiel $^{1,2,11 \bowtie}$
}

Within the past decade, we have seen outbreaks of numerous viruses, including Middle East respiratory syndrome coronavirus (MERS-CoV) ${ }^{5}$, ZIKA virus $^{6}$, Ebola virus ${ }^{7}$ and, at the end of 2019, SARS-CoV-2- which was first detected in Wuhan, Hubei province, $\mathrm{China}^{4}$, but rapidly developed into a pandemic. During the early phase of the SARS-CoV-2 outbreak, virus isolates were not available to health authorities and the scientific community, even though these isolates are urgently needed to generate diagnostic tools, to develop and assess antivirals and vaccines, and to establish appropriate in vivo models. The generation of the SARS-CoV-2 from chemically synthesized DNA could bypass the limited availability of virus isolates and would furthermore enable genetic modifications and functional characterization. However, although E. coli proved very useful for the cloning of many viral genomes, it has a number of disadvantages when used for the assembly and stable maintenance of full-length molecular clones of emerging RNA viruses, including coronaviruses.
Synthetic genomics is a field fuelled by the efforts to create a bacterial cell that is controlled by a synthetic genome ${ }^{8}$. Genome-wide reassembly of the approximately 1.1-megabase (Mb) genome of Mycoplasma was first attempted using $E$. coli as an intermediate host $^{8}$; however, the maintenance of 100-kilobase (kb) DNA fragments appeared to be very difficult in this host. Therefore, the yeast $S$. cerevisiae was chosen to clone, assemble and mutagenize entire Mycoplasma genomes ${ }^{9,10}$. The rationale for using a yeast cloning system is the ability of yeast to recombine overlapping DNA fragments in vivo, which led to the development of a technique called transformation-associated recombination (TAR) cloning ${ }^{11}$.

More recently ${ }^{12,13}$, TAR cloning was successfully used for the assembly, genetic engineering and rescue of large DNA viruses such as cytomegalovirus and herpes simplex virus 1 . For coronaviruses that belong to a family of positive-stranded RNA viruses termed Coronaviridae, the generation of full-length molecular clones has long been hampered by

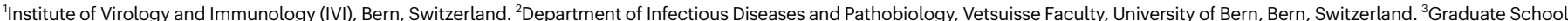
for Biomedical Science, University of Bern, Bern, Switzerland. ${ }^{4}$ Institute of Veterinary Bacteriology, Vetsuisse Faculty, University of Bern, Bern, Switzerland. Insitute for Infectious Diseases, University of Bern, Bern, Switzerland. ${ }^{6}$ Department for Molecular and Medical Virology, Ruhr-Universität Bochum, Bochum, Germany. ${ }^{7}$ Institute of Virology, Charité-Universitätsmedizin Berlin,

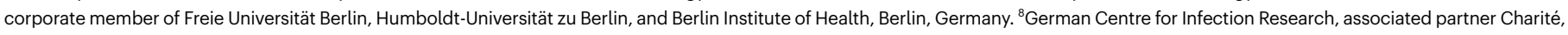

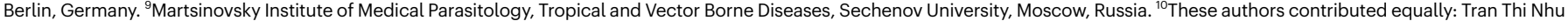

Thao, Fabien Labroussaa, Nadine Ebert. ${ }^{11}$ These authors jointly supervised this work: Joerg Jores, Volker Thiel. ${ }^{\bowtie}$ e-mail: joerg.jores@vetsuisse.unibe.ch; volker.thiel@vetsuisse.unibe.ch 


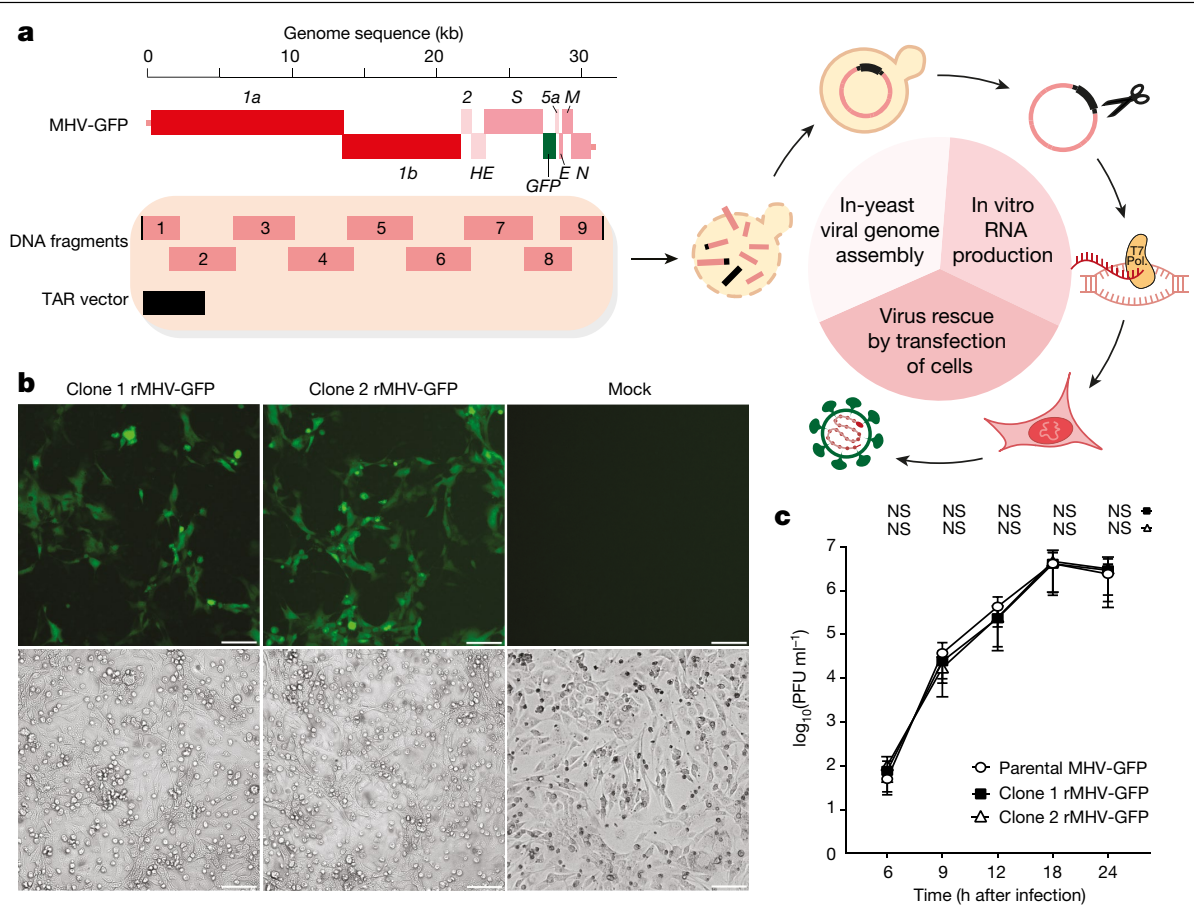

Fig. 1 | Application of yeast-based TAR cloning to generate viral cDNA clones and the recovery of recombinant MHV-GFP. a, General workflow of TAR cloning and virus rescue. In-yeast genome reconstruction requires one-step delivery of overlapping DNA fragments that cover the viral genome and a TAR vector in yeast. Viral ORFs and the ORF for GFP are indicated. Transformed DNA fragments are assembled by homologous recombination in yeast to generate a YAC that contains the full-length viral cDNA sequence. In vitro production of infectious capped viral RNA starts with the isolation of the YAC, followed by plasmid linearization to provide a DNA template for run-off T7 RNA polymerase-based transcription. Virus rescue is initiated by electroporation of BHK-MHV-N cells, after which virus production and amplification is carried out by culturing the virus with susceptible cells. b, Recovery of infectious rMHV-GFP from yeast clones 1 and 2 . Cell-culture supernatants-which contain viruses produced after virus rescue of two MHV-GFP YAC clones-were used to infect $17 \mathrm{Cl}-1$ cells. At $48 \mathrm{~h}$ after infection, infected cells were visualized for GFP expression (top) and by bright-field microscopy (bottom). Mock represents $17 \mathrm{Cl}-1$ cells inoculated with the supernatant from BHK-MHV-N cells electroporated without viral RNAs. Images are representative of two independent experiments. Scale bars, $100 \mu \mathrm{m}$. c, Replication kinetics of parental MHV-GFP and rMHV-GFP clones 1 and 2. L929 cells were infected (multiplicity of infection $(\mathrm{MOI})=0.1$ ), and cell-culture supernatants were collected at the indicated time points after infection and titrated by plaque assay. PFU, plaque forming units. Data represent the mean \pm s.d. of three independent biological experiments $(n=3)$. Statistical significance was determined by two-sided unpaired Student's $t$-test without adjustments for multiple comparisons. NS, not significant. $P$ values (from left to right): top, NS, $P=0.2905$; NS, $P=0.3504$; NS, $P=0.1817$; NS, $P=0.9862$; NS, $P=0.6738$; bottom, NS, $P=0.0835$; NS,$P=0.1400 ; \mathrm{NS}, P=0.2206$; NS, $P=0.8020 ; \mathrm{NS}, P=0.5894$.

(strain VL6-48N), and the resulting clones were screened for the correct assembly of the yeast artificial chromosome (YAC) containing the cloned MHV genome by multiplex PCRs that covered the junctions between recombined fragments. This screen revealed that more than $90 \%$ of the clones tested were positive, indicating that the assembly in yeast is highly efficient (Supplementary Fig. 1a). To rescue MHV-GFP, we randomly chose two clones, purified and linearized the YACs using Pacl (Extended Data Table 1) and subjected the YACs to in vitro transcription using T7 RNA polymerase to generate capped viral genomic RNA. This RNA was transfected together with an in vitro-transcribed mRNA that encodes the MHV nucleocapsid (N) protein into BHK-MHV-N cells, which were then mixed with MHV-susceptible $17 \mathrm{Cl}-1$ cells as previously described $^{14}$. Cytopathogenic effects, virus-induced syncytia and GFP-expressing cells were readily detectable for both clones within $48 \mathrm{~h}$, indicating the successful recovery of infectious virus (Fig. 1b). Finally, we assessed the replication kinetics of the recovered viruses, which were indistinguishable from the parental MHV-GFP line (Fig.1c).

To address whether the synthetic genomics platform can be applied to other coronaviruses and whether it can be used for rapid mutagenesis, we used a molecular BAC clone of MERS-CoV ${ }^{16}$. We PCR-amplified eight overlapping DNA fragments that covered the MERS-CoV genome (Extended Data Fig. 1a, Supplementary Fig. 1b and Supplementary Table 1). The 5' - and 3'-terminal fragments contained the T7 RNA polymerase promoter upstream of the MERS-CoV 5' end and the restriction endonuclease cleavage site Mlul downstream of the poly(A) sequence, 
Table 1| RNA virus genomes cloned using the synthetic genomics platform

\begin{tabular}{|c|c|c|c|c|c|c|}
\hline Virus & Family & $\begin{array}{l}\text { Size } \\
\text { (kb) }\end{array}$ & Template & $\begin{array}{l}\text { Fragment } \\
\text { generation }\end{array}$ & $\begin{array}{l}\text { Number of } \\
\text { fragments }\end{array}$ & Virus rescue \\
\hline MHV-GFP & Coronaviridae & 31.9 & Viral RNA, DNA clone & RT-PCR, PCR & 9 & Yes \\
\hline MERS-CoV & Coronaviridae & 30.1 & DNA clone & PCR & 8 & Yes \\
\hline MERS-CoV-GFP & Coronaviridae & 30.7 & $\begin{array}{c}\text { DNA clone, GFP plasmid } \\
\text { DNA }\end{array}$ & PCR & 10 & Yes \\
\hline HCoV-229E & Coronaviridae & 27.3 & Viral RNA, DNA clone & RT-PCR, PCR & 13 & Not attempted \\
\hline HCoV-HKU1 & Coronaviridae & 29.9 & $\begin{array}{c}\text { Synthetic DNA, viral } \\
\text { RNA }\end{array}$ & PCR, RT-PCR & 11 & Not attempted \\
\hline MERS-CoV Riyadh-1734-2015 & Coronaviridae & 30 & Viral RNA & RT-PCR & 8 & Not attempted \\
\hline ZIKA virus & Flaviviridae & 10.8 & Viral RNA & RT-PCR & 6 & Not attempted \\
\hline Human RSV-B & Pneumoviridae & 15 & Clinical sample & RT-PCR & 4 & Not attempted \\
\hline SARS-CoV-2 & Coronaviridae & 30 & $\begin{array}{c}\text { Synthetic DNA, viral } \\
\text { RNA }\end{array}$ & Plasmid, RT-PCR & 12 & Yes \\
\hline SARS-CoV-2-GFP & Coronaviridae & 30.5 & $\begin{array}{c}\text { Synthetic DNA, viral } \\
\text { RNA }\end{array}$ & $\begin{array}{c}\text { Plasmid, RT-PCR/ } \\
\text { PCR }\end{array}$ & 14 & Yes \\
\hline synSARS-CoV-2-GFP & Coronaviridae & 30.5 & Synthetic DNA & Plasmid, PCR & 19 & Yes \\
\hline
\end{tabular}

The number of fragments excludes the TAR vector fragment.

and overlapping sequences with the TAR plasmid pVC604. To mutagenize the MERS-CoV clone, fragment 7 was divided into three overlapping PCR fragments to place the GFP gene in frame with a porcine teschovirus $2 \mathrm{~A}$ element and open-reading frame $4 \mathrm{a}(\mathrm{ORF} 4 \mathrm{a})^{16}$ (Extended Data Fig. 1a and Supplementary Table 1). Again, almost all YAC clones were successfully assembled (Supplementary Fig. 1b, c). Virus rescue from cloned DNA was performed as described previously ${ }^{16}$, resulting in recombinant ( $r$ )MERS-CoV and rMERS-CoV-GFP (Extended Data Fig. 1b). This demonstrates that the synthetic genomics platform is suitable to genetically modify coronavirus genomes. As expected, the replication kinetics of rMERS-CoV and rMERS-CoV-GFP were slightly reduced compared with the cell-culture-adapted MERS-CoV-EMC strain (Extended Data Fig. 1c).

Next, we thoroughly evaluated the stability of the cloned genomes, the range of applicability to other virus genomes and whether molecular clones can be generated from clinical samples. Yeast clones that contained YACs encoding MHV-GFP and MERS-CoV were passaged 15-17 times, and sequencing revealed that the genomes could be stably maintained (Extended Data Table 2). We further cloned several other coronaviruses (HCoV-229E² ${ }^{2}$ HCoV-HKU1(GenBank: NC_006577) and MERS-CoV-Riyadh-1734-2015 (GenBank: MN481979)) and viruses of other families, such as ZIKA virus (family Flaviviridae, GenBank: KX377337) and human respiratory syncytial virus (hRSV; family Pneumoviridae) (Table1), which are known to be difficult to clone and stably maintain in E. coli. As shown in Supplementary Fig. 1d-h, cloning of these viral genomes in yeast was in all cases successful irrespectively of the virus source, the nucleic acid template or the number of DNA fragments. Of note, we cloned hRSV-B without any prior information on the virus genotype directly from a clinical sample (nasopharyngeal aspirate) by designing RSV consensus primers to amplify four overlapping DNA fragments (Supplementary Table 1) (sequence submitted to GenBank: MT107528). Collectively, these results demonstrate that the synthetic genomics platform provides the technical advance to rapidly generate molecular clones of diverse RNA viruses by using virus isolates, cloned DNA, synthetic DNA or clinical samples as starting material.

The detection of a new coronavirus in China at the end of 2019 prompted us to test the applicability of our synthetic genomics platform to reconstruct the virus based on the genome sequences released on 10-11 January 2020 (Fig. 2). We divided the genome into 12 overlapping DNA fragments (Fig. 3a, Extended Data Table 3, Supplementary Fig. $1 \mathrm{i}$ and Supplementary Table 1). In parallel, we aimed to generate a SARS-CoV-2 clone that expressed GFP, as this could facilitate the screening of antiviral compounds and be used to establish diagnostic assays (for example, virus neutralization assays). This was achieved by dividing fragment 11 into three subfragments (Fig. 3a, Supplementary Fig. $1 \mathrm{j}$ and Supplementary Table 1), and GFP was inserted in-frame of ORF7a, replacing nucleotides $40-282$. We noticed that nucleotides 3-5 at the 5 ' end of the reported SARS-CoV-2 sequence (5'-AUUAAAGG; GenBank MN996528.1; nucleotides that are different are highlighted in bold) differed from SARS-CoV (5'-AUAUUAGG; GenBank AY291315) and from the more closely related bat SARS-related CoVs ZXC21 and ZC45 (5'-AUAUUAGG) ${ }^{4,17,18}$ (Extended Data Fig. 2a, b). We therefore designed three $5^{\prime}$-end versions, and each version was combined with the remaining SARS-CoV-2 genome (constructs 1-3) or a corresponding SARS-CoV-2-GFP genome (constructs 4-6). Constructs 1 and 4 contained the $5^{\prime}$ end modified by three nucleotides according to the bat SARS-related CoVs ( $5^{\prime}$-AUAUUAGG), constructs 2 and 5 contained the $1245^{\prime}$-terminal nucleotides of SARS-CoV, and constructs 3 and 6 contained the reported SARS-CoV-2 sequence (5'-AUUAAAGG; according to MN996528.1) (Extended Data Fig. 2a, b). Notably, differences between SARS-CoV-2 and SARS-CoV within the $5^{\prime}$-terminal 124 nucleotides are in agreement with the predicted RNA secondary structures (Extended Data Fig. 2b).

Fourteen synthetic DNA fragments were ordered as sequence-confirmed plasmids and all but fragments 5 and 7 were delivered (Extended Data Table 3, Supplementary Data 1). As we received SARS-CoV-2 viral RNA from an isolate of a Munich patient (BetaCoV/Germany/BavPat1/2020) at the same time, we amplified the regions of fragments 5 and 7 by RT-PCR (Supplementary Table 1 ). TAR cloning was immediately initiated, and for all six SARS-CoV-2 and SARS-CoV-2-GFP constructs we obtained correctly assembled molecular clones (Extended Data Fig. 3a and Supplementary Fig. 1i,j). Because sequence verification was not possible within this short time frame, we randomly selected two clones for each construct (Extended Data Fig. 3a), isolated the YAC DNA and performed in vitro transcription. The resulting RNAs were electroporated together with an mRNA that encodes the SARS-CoV-2 N protein into BHK-21 and, in parallel, into BHK-SARS-N cells that expressed the SARS-CoV N protein ${ }^{19}$ (Extended Data Fig. 3b). Electroporated cells were seeded on Vero E6 cells and two days later we observed green fluorescent signals in cells that received the GFP-encoding SARS-CoV-2 RNAs. Indeed, we could rescue infectious viruses for almost all rSARS-CoV-2 and rSARS-CoV-2-GFP clones (Extended Data Fig. 3b). As shown in Fig. 3b, for rSARS-CoV-2 clones 


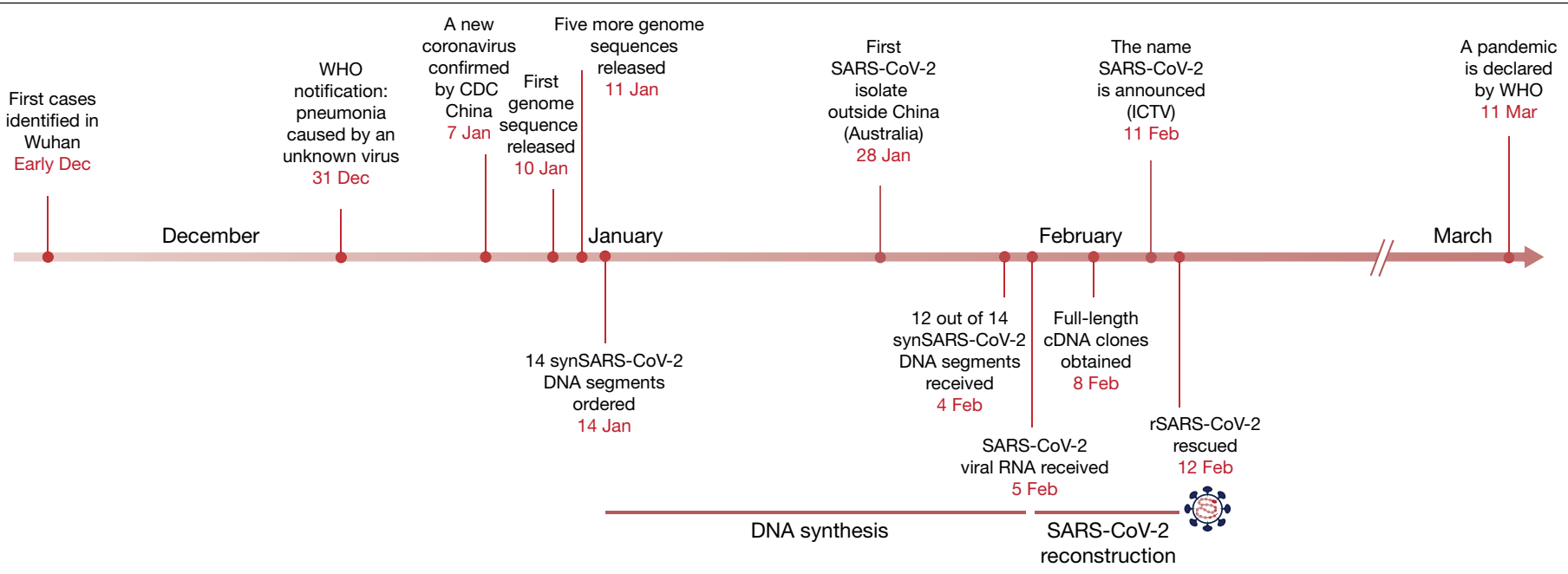

Fig. 2 Timeline of the reconstruction and recovery of rSARS-CoV-2 in relation to key events of the COVID-19 pandemic. Illustration of the rapidity of rSARS-CoV-2 reconstruction along with the timeline of key events of the
COVID-19 pandemic. CDC, Center for Disease Control and Prevention; ICTV, International Committee on Taxonomy of Viruses; WHO, World Health Organization.
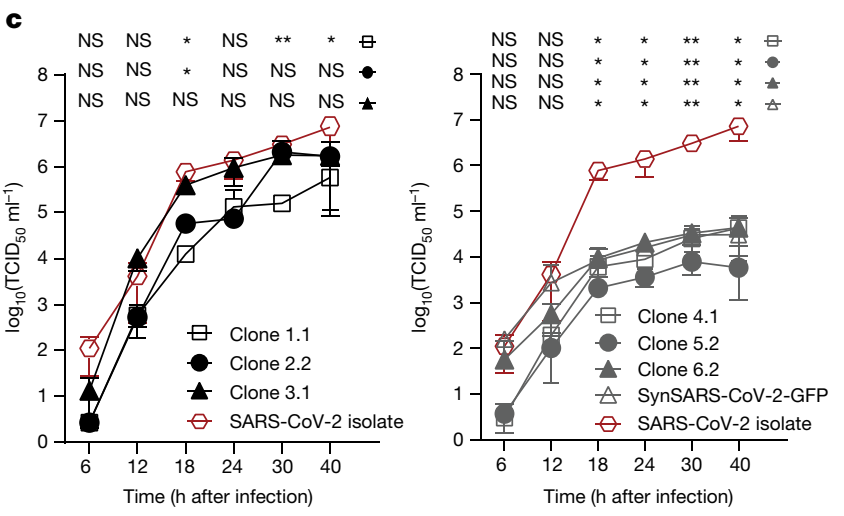

a Genome sequence $(\mathrm{kb})$
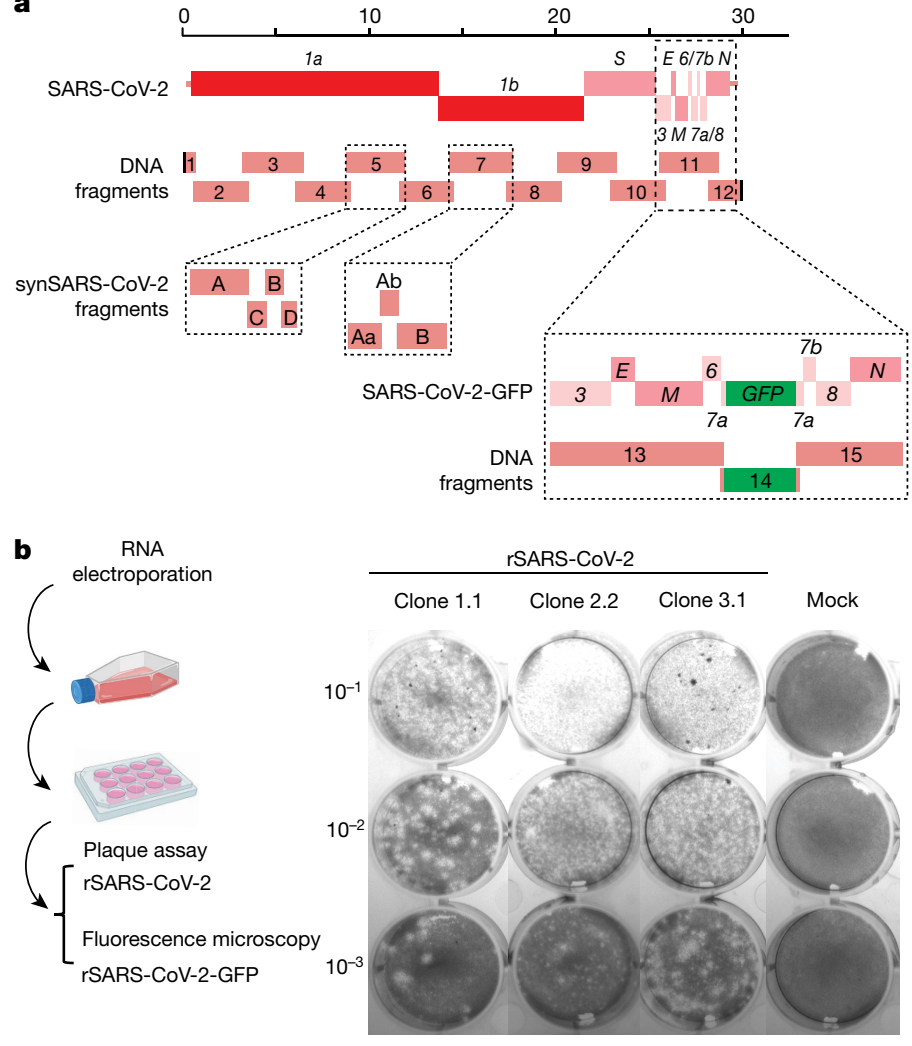

Fig. 3 | Reconstruction, rescue and characterization of rSARS-CoV-2, rSARS-CoV-2-GFP and synSARS-CoV-2-GFP. a, Schematic representation of the SARS-CoV-2 genome organization and DNA fragments used to clone rSARS-CoV-2, rSARS-CoV-2-GFP and synSARS-CoV-2-GFP. Inserts show synthetic subfragments comprising fragments 5 (A-D) and 7 (Aa, Ab, B), and the fragments used to insert the GFP gene (fragments 13-15). b, Left, schematic of the experiment. Middle, rescue of rSARS-CoV-2 from yeast clones 1.1, 2.2 and 3.1. Supernatants $\left(10^{-1}, 10^{-2}\right.$ and $\left.10^{-3} \mathrm{ml}\right)$ of cells infected with the indicated clones or mock-infected cells were transferred to Vero E6 cells to detect plaques (rSARS-CoV-2). Right, rescue of rSARS-CoV-2-GFP from yeast clones 4.1, 5.2 and 6.2. Supernatants $(1 \mathrm{ml})$ from individual rescue experiments were transferred to Vero E6 cells to detect green fluorescence (rSARS-CoV-2-GFP). Mock, uninfected cells. Scale bars, $100 \mu \mathrm{m}$.c, Replication kinetics of rSARS-CoV-2 clones 1.1, 2.2, 3.1 (left) and rSARS-CoV-2-GFP clones 4.1, 5.2, 6.2 and synSARS-CoV-2-GFP (right) compared with the SARS-CoV-2 isolate. Vero E6

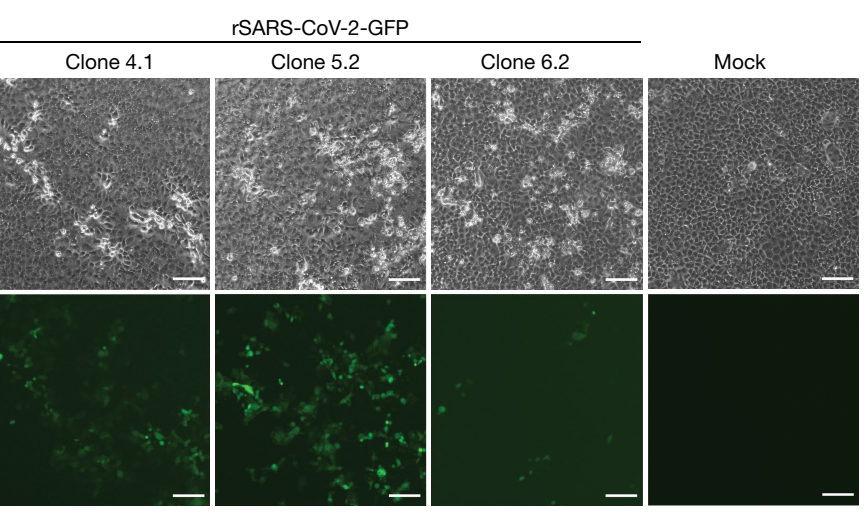

cells were infected $(\mathrm{MOI}=0.01)$, and supernatants were collected at the indicated time points after infection and titrated (50\% tissue culture infectious dose $\left(\mathrm{TCID}_{50}\right)$ assay). Data represent the mean \pm s.d. of three independent biological replicates. Statistical significance was determined for each clone against the SARS-CoV-2 isolate by two-sided unpaired Student's $t$-test without adjustments for multiple comparisons. $P$ values (from left to right): left, top, NS, $P=0.0851$; NS,$P=0.1775 ;{ }^{*} P=0.0107 ; \mathrm{NS}, P=0.0648 ; * * P=0.0013$; ${ }^{*} P=0.0373$; middle, NS, $P=0.0851$; NS, $P=0.1713 ;{ }^{*} P=0.0133$; NS, $P=0.0535$; NS, $P=0.0909$; NS, $P=0.0632$; bottom, NS, $P=0.1119$; NS, $P=0.1641$; NS,$P=0.0994$; NS, $P=0.4921$; NS,$P=0.3336$; NS, $P=0.0790$; right, top, NS, $P=0.0858$; NS, $P=0.1429 ;{ }^{*} P=0.0104 ;{ }^{*} P=0.0466 ;{ }^{* *} P=0.0011 ;{ }^{*} P=0.0287$; second, NS, $P=0.0872$; NS,$P=1360 ;{ }^{*} P=0.0102 ;{ }^{*} P=0.0461 ;{ }^{* *} P=0.0011 ;{ }^{*} P=0.0282$; third, NS, $P=0.4810$; NS,$P=0.1758 ;{ }^{*} P=0.0106 ;{ }^{*} P=0.0478 ;{ }^{* *} P=0.0011 ; * P=0.0287$; bottom, NS, $P=0.3739 ; \mathrm{NS}, P=0.6817 ;{ }^{*} P=0.0106 ;{ }^{*} P=0.0473 ;{ }^{* *} P=0.0011$ ${ }^{*} P=0.0285$. 
1.1, 2.2, and 3.1, plaques were readily detectable, demonstrating that infectious virus has been recovered irrespectively of the $5^{\prime}$-terminal sequences. Sequencing of the YACs and corresponding rescued viruses revealed that almost all DNA clones and viruses contained the correct sequence, except for some individual clones that contained mutations within fragments 5 and 7 that were probably introduced by RT-PCR (Extended Data Table 4). Nevertheless, we obtained at least one correct YAC clone for all constructs except for construct 6 . To correct this, we reassembled construct 6 by replacing the RT-PCR-generated fragments 5 and 7 with four and three shorter synthetic double-stranded (ds)DNA fragments, respectively. The resulting molecular clone was used to rescue the synthetic SARS-CoV-2-GFP (synSARS-CoV-2-GFP) virus without any mutations exclusively from chemically synthesized DNA (Extended Data Fig. 4 and Extended Data Tables 3, 4).

Next we assessed the $5^{\prime}$ end of the recombinant viruses and the Munich virus isolate and confirmed the published 5 ' end sequence of SARS-CoV-2 (5'-AUUAAAGG; GenBank MN996528.3). Full-length sequencing of the viral genomes and $5^{\prime}$ rapid amplification of cDNA end (5'-RACE) analysis of the recombinant viruses confirmed the identity of each virus, and showed that the $5^{\prime}$ end variant of each virus retained the cloned 5' terminus (Extended Data Fig. 2a). This demonstrates that the $5^{\prime}$ ends of SARS-CoV and bat SARS-related CoVs ZXC21 and ZC45 are compatible with the replication machinery of SARS-CoV-2. Sequencing results also revealed the identity of leader-body junctions of SARS-CoV-2 subgenomic mRNAs, which are identical to those of SARS-CoV ${ }^{18}$ (Extended Data Fig. 2c-h). We also analysed rSARS-CoV-2 clone 3.1 for protein expression and demonstrated the presence of the SARS-CoV-2 nucleocapsid protein in dsRNA-positive cells (Extended Data Fig. 5b). The replication kinetics of rSARS-CoV-2 clone 3.1, which contains the authentic $5^{\prime}$ terminus, was indistinguishable from replication of the SARS-CoV-2 isolate, while clones 1.1 and 2.2 showed slightly reduced replication (Fig. $3 \mathrm{c}$, left). All rSARS-CoV-GFP clones and synSARS-CoV-GFP displayed similar growth kinetics but they were significantly reduced compared with the SARS-CoV-2 isolate, suggesting that the insertion of GFP and/or the partial deletion of ORF7a affects replication (Fig. 3c, right and Extended Data Fig. 5d-f). Despite the reduced replication, green fluorescence was readily detectable and we demonstrated the use of the synSARS-CoV-GFP clone for antiviral drug screening by testing remdesivir, a promising compound for the treatment of COVID-19 ${ }^{20}$ (Extended Data Fig. 5c). Similarly, the simple readout of green fluorescence greatly facilitates the demonstration of virus neutralization with human serum (Extended Data Fig. 5a).

Our results demonstrate the full functionality of the SARS-CoV-2 reverse-genetics system and we expect that this fast, robust and versatile synthetic genomics platform will provide new insights into the molecular biology and pathogenesis of a number of emerging RNA viruses. Although homologous recombination in yeast has already been used for the generation of a number of molecular virus clones in the past ${ }^{12,13,21,22}$, we present a thorough evaluation of the feasibility of this approach to rapidly generate full-length cDNAs for large RNA viruses that have a known history of instability in $E$. coli. We show that one main advantage of the TAR cloning system is that the viral genomes can be fragmented to at least 19 overlapping fragments and reassembled with remarkable efficacy. This facilitated the cloning and rescue of rSARS-CoV-2 and rSARS-CoV-2-GFP within one week. It should be noted that we see considerable potential to reduce the time of DNA synthesis. Currently, synthetic DNA fragments get routinely cloned in $E$. coli, which turned out to be problematic for SARS-CoV-2 fragments 5 and 7 . We, however, used shorter synthetic dsDNA parts to assemble these fragments by TAR cloning and to generate the molecular clone synSARS-CoV-2-GFP by using exclusively chemically synthesized DNA, which is an additional proof of the superior cloning efficiency of yeast- versus $E$. coli-based systems.

The COVID-19 pandemic emphasizes the need for preparedness to rapidly respond to emerging virus threats. The rapidity of our synthetic genomics approach to generate SARS-CoV-2 and the applicability to other emerging RNA viruses make this system an attractive alternative to provide infectious virus samples to health authorities and diagnostic laboratories without the need of having access to clinical samples. As the COVID-19 pandemic is ongoing, we expect to see sequence variations and possibly phenotypic changes of the evolving SARS-CoV-2 virus in the human host. With this synthetic genomics platform, it is now possible to rapidly introduce such sequence variations into the infectious clone and to functionally characterize SARS-CoV-2 evolution in real time.

\section{Online content}

Any methods, additional references, Nature Research reporting summaries, source data, extended data, supplementary information, acknowledgements, peer review information; details of author contributions and competing interests; and statements of data and code availability are available at https://doi.org/10.1038/s41586-020-2294-9.

1. Almazán, F. et al. Engineering the largest RNA virus genome as an infectious bacterial artificial chromosome. Proc. Natl Acad. Sci. USA 97, 5516-5521 (2000).

2. Thiel, V., Herold, J., Schelle, B. \& Siddell, S. G. Infectious RNA transcribed in vitro from a cDNA copy of the human coronavirus genome cloned in vaccinia virus. J. Gen. Virol. 82, 1273-1281 (2001).

3. Yount, B., Curtis, K. M. \& Baric, R. S. Strategy for systematic assembly of large RNA and DNA genomes: transmissible gastroenteritis virus model. J. Virol. 74, 10600-10611 (2000).

4. Zhu, N. et al. A novel coronavirus from patients with pneumonia in China, 2019. N. Engl. J. Med. 382, 727-733 (2020).

5. Zaki, A. M., van Boheemen, S., Bestebroer, T. M., Osterhaus, A. D. \& Fouchier, R. A. Isolation of a novel coronavirus from a man with pneumonia in Saudi Arabia. N. Engl. J. Med. 367, 1814-1820 (2012).

6. Cao-Lormeau, V. M. et al. Zika virus, French Polynesia, South Pacific, 2013. Emerg. Infect. Dis. 20, 1084-1086 (2014).

7. Baize, S. et al. Emergence of Zaire Ebola virus disease in Guinea. N. Engl. J. Med. 371, 1418-1425 (2014).

8. Gibson, D. G. et al. Creation of a bacterial cell controlled by a chemically synthesized genome. Science 329, 52-56 (2010).

9. Lartigue, C. et al. Creating bacterial strains from genomes that have been cloned and engineered in yeast. Science 325, 1693-1696 (2009).

10. Benders, G. A. et al. Cloning whole bacterial genomes in yeast. Nucleic Acids Res. $\mathbf{3 8}$, 2558-2569 (2010)

11. Kouprina, N. \& Larionov, V. Selective isolation of genomic loci from complex genomes by transformation-associated recombination cloning in the yeast Saccharomyces cerevisiae. Nat. Protoc. 3, 371-377 (2008).

12. Vashee, S. et al. Cloning, assembly, and modification of the primary human cytomegalovirus isolate Toledo by yeast-based transformation-associated recombination. mSphere 2, e00331-17 (2017).

13. Oldfield, L. M. et al. Genome-wide engineering of an infectious clone of herpes simplex virus type 1 using synthetic genomics assembly methods. Proc. Natl Acad. Sci. USA 114, E8885-E8894 (2017).

14. Coley, S. E. et al. Recombinant mouse hepatitis virus strain A59 from cloned full-length cDNA replicates to high titers in vitro and is fully pathogenic in vivo. J. Virol. 79, 3097-3106 (2005).

15. Züst, R. et al. Coronavirus non-structural protein 1 is a major pathogenicity factor: implications for the rational design of coronavirus vaccines. PLoS Pathog. 3, e109 (2007).

16. Muth, D et al. Transgene expression in the genome of Middle East respiratory syndrome coronavirus based on a novel reverse genetics system utilizing Red-mediated recombination cloning. J. Gen. Virol. 98, 2461-2469 (2017).

17. Hu, D. et al. Genomic characterization and infectivity of a novel SARS-like coronavirus in Chinese bats. Emerg. Microbes Infect. 7, 1-10 (2018).

18. Thiel, V. et al. Mechanisms and enzymes involved in SARS coronavirus genome expression. J. Gen. Virol. 84, 2305-2315 (2003).

19. van den Worm, S. H. et al. Reverse genetics of SARS-related coronavirus using vaccinia virus-based recombination. PLoS ONE 7, e32857 (2012).

20. Sheahan, T. P. et al. Comparative therapeutic efficacy of remdesivir and combination lopinavir, ritonavir, and interferon beta against MERS-CoV. Nat. Commun. 11, 222 (2020).

21. Polo, S., Ketner, G., Levis, R. \& Falgout, B. Infectious RNA transcripts from full-length dengue virus type 2 cDNA clones made in yeast. J. Virol. 71, 5366-5374 (1997).

22. Nikiforuk, A. M. et al. Rapid one-step construction of a Middle East respiratory syndrome (MERS-CoV) infectious clone system by homologous recombination. J. Virol. Methods 236, 178-183 (2016).

Publisher's note Springer Nature remains neutral with regard to jurisdictional claims in published maps and institutional affiliations.

(c) The Author(s), under exclusive licence to Springer Nature Limited 2020 


\section{Methods}

\section{Cells and general culture conditions}

Vero, Vero B4 and Vero B6 cells (all ATCC) were cultured in Dulbecco's modified Eagle's medium (DMEM); BHK-21, BHK-MHV-N (BHK-21 cells expressing the N protein of MHV strain A59) ${ }^{14}$, BHK-SARS-N (BHK-21 cells expressing the $\mathrm{N}$ protein of SARS $)^{19}, \mathrm{Huh}-7^{23}, \mathrm{~L}$ (929 ${ }^{23}$ and mouse $17 \mathrm{Cl}-1^{23}$ cells were grown in minimal essential medium (MEM). Both types of medium were supplemented with $10 \%$ fetal bovine serum, $1 \times$ non-essential amino acids, 100 units $\mathrm{ml}^{-1}$ penicillin and $100 \mathrm{\mu g} \mathrm{ml}^{-1}$ streptomycin. BHK-SARS-N cells were grown using MEM supplemented with $5 \%$ fetal bovine serum, $1 \times$ non-essential amino acids, 100 units $\mathrm{ml}^{-1}$ penicillin, and $100 \mathrm{\mu g} \mathrm{ml}^{-1}$ streptomycin, $500 \mathrm{\mu g} \mathrm{ml}^{-1} \mathrm{G} 418$ and $10 \mu \mathrm{g} \mathrm{ml}^{-1}$ puromycin. BHK-MHV-N and BHK-SARS-N were treated with $1 \mu \mathrm{g} \mathrm{ml}^{-1}$ doxycyclin $24 \mathrm{~h}$ before electroporation. All cells were maintained at $37^{\circ} \mathrm{C}$ and in a $5 \% \mathrm{CO}_{2}$ atmosphere.

\section{Cultured viruses}

MHV-GFP ${ }^{14,15}$ and HCoV-229E ${ }^{2}$ were cultured in mouse $17 \mathrm{Cl}-1$ and human Huh-7 cells, respectively. MERS-CoV-EMC ${ }^{24}$ was cultured in Vero B4 cells. HCoV-HKU1 strain Caen-1 (GenBank: NC_006577) was cultured in human airway epithelial cultures ${ }^{25}$. ZIKA virus strain PRVABC-59 (GenBank:KX377337) was provided by M. Alves and was cultured in Vero cells. SARS-CoV-2 (SARS-CoV-2/München-1.1/2020/929) was cultured in Vero E6 cells.

\section{Bacterial and yeast strains}

E. coli DH5 $\alpha$ (Thermo Scientific) and TransforMax Epi300 (Epicentre) were used to propagate the pVC604 and pCC1BAC-His3 TAR vectors ${ }^{8}$, respectively. The bacteria were grown in lysogeny broth medium supplemented with the appropriate antibiotics at $37^{\circ} \mathrm{C}$ overnight. E. coli Epi300 cells containing the different synthetic fragments of SARS-CoV-2 in pUC57 or pUC57mini were grown at $30^{\circ} \mathrm{C}$ to decrease the risk of instability and/or toxicity. Saccharomyces cerevisiae VL6-48N (MAT $\alpha$ trp1- $\Delta 1$ ura3- $\Delta 1$ ade2-101 his3- $\Delta 200$ lys 2 met14 cir $^{\circ}$ ) was used for all yeast transformation experiments ${ }^{26}$. Yeast cells were first grown in YPDA broth (Takara Bio), and transformed cells were plated on minimal synthetic defined (SD) agar without histidine (SD-His) (Takara Bio). S. cerevisiae VL6-48N-derived clones carrying different YACs were never streaked out together on the same agar dishes as mating switching and resulting recombination might occur at a very low frequency.

\section{Generation of viral subgenomic fragments for TAR cloning using} viral RNA, infectious cDNA clones and synthetic DNA

Table 1 displays the templates used to clone the different viral genomes into $S$. cerevisiae. In general, viral DNA fragments were obtained by RT-PCR of viral RNA extracted from viral strains, isolates and from clinical specimens, using the SuperScript IV One-Step RT-PCR System following the manufacturer's instructions. Additionally, some fragments were PCR-amplified from vaccinia virus-cloned cDNA ${ }^{2,14}$, BAC-cloned cDNA ${ }^{16}$ and plasmid-cloned synthetic DNA (GenScript), using the CloneAmp HiFi PCR Premix according to the manufacturer's instructions. Accessory sequences, that is, enhanced GFP and porcine teschovirus-12A (P2A) for the MERS-CoV-GFP construct, TurboGFP for SARS-CoV-2-GFP and T7 RNA polymerase promoter-hammerhead ribozyme and ribozyme-T7 terminator for human RSV-B, were amplified from plasmids.

For all coronaviruses, the fragment encompassing the viral 5 ' untranslated regions (UTR) contained the T7 RNA polymerase promoter sequence immediately upstream of the $5^{\prime}$ end of the genome, and the fragment encompassing the ${ }^{\prime}$ ' end of the genome contained a unique restriction site (Extended Data Table1) downstream of the poly(A) tail.

HCoV-HKU1 synthetic fragments 1-4 were provided individually cloned into pUC57 by GenScript (Extended Data Table 3). MERS-CoV-Riyadh-1734-2015 (GenBank: MN481979) fragments 1-8 were synthesized and cloned into pUC57 by GenScript (Extended Data Table 3), containing homologous regions to TAR vectors pVC604 and pCC1BAC-His3. Similarly, synthetic ZIKA virus fragment 6 cloned into pUC57 contained a hepatitis delta virus ribozyme sequence and pCC1BAC-his3 homology downstream of the viral 3' UTR (Extended Data Table 3).

The SARS-CoV-2 synthetic DNA fragments were delivered cloned into pUC57 or pUC57mini by GenScript (Supplementary Data 1, Extended Data Table 3). Fragments 1.1, 1.2,1.3 and 12 contained homologous sequences to pCC1BAC-His3. Each fragment was sequence verified using Sanger sequencing after plasmid isolation using QIAGEN Midiprep kit (QIAGEN). Fragments were released from the vector using the restriction enzymes described in Extended Data Table 3. Restricted fragments were subsequently gel-purified using standard methods ${ }^{27}$. DNA concentrations and purities of all fragments to be used for TAR cloning were determined using NanoDrop 2000/2000c Spectrophotometer (Thermo Scientific).

\section{In-yeast cloning of viral genomes using TAR}

In general, we used overlapping DNA fragments for TAR cloning with overlaps ranging from 45 to $500 \mathrm{bp}$. As all of our cloning experiments worked well, we did not assess whether the lengths of the overlap affected homologous recombination efficacy. The vectors pVC604 ${ }^{11}$ and pCC1BAC-His $3^{8}$ were used for TAR cloning. These vectors were amplified by PCR using primers containing at least 45-bp overlaps to fragments encompassing the 5' or 3' ends of different viral genomes (Supplementary Table 1). Amplification was performed using KOD Hot Start DNA polymerase (Merck Millipore) according to the manufacturer's instructions. Templates used for generating fragments for TAR cloning are shown in Table 1. TAR cloning was also used to reconstruct the full-length synthetic fragments 5 and 7 in yeast (Extended Data Fig. 4b, c).

Yeast transformation was done using the high-efficiency lithium acetate/SS carrier DNA/PEG method as described elsewhere ${ }^{28}$. In brief, yeast cells were grown in rich YPDA medium (Takara Bio) at $30^{\circ} \mathrm{C}$ with agitation until an optical density at $600 \mathrm{~nm}$ of 1.0 was reached. Then, $3 \mathrm{ml}$ of yeast culture was used per transformation event. DNA mixtures were prepared beforehand and contained 100-200 fmol of $3^{\prime}$ and 5' open ends for all fragments. Transformation mixtures were plated onto SD-His plates (Takara Bio) and incubated at $30^{\circ} \mathrm{C}$ for $48 \mathrm{~h}$. Colonies were resuspended in $20 \mu \mathrm{l}$ of SD-His broth, and DNA was extracted following the GC prep method ${ }^{29}$. Extracted DNA was used as template for screening by multiplex PCR using the QIAGEN Multiplex PCR kit (QIAGEN) according to the manufacturer's instruction. One or two multiplex PCRs were designed to encompass different subsets of primer pairs, and cover all desired recombination junctions (Supplementary Table 1). Clones tested positive for all junctions were grown in SD-His until late logarithmic phase, and plasmids were extracted from $500 \mathrm{ml}$ culture using the QIAGEN Maxiprep Kit (QIAGEN) with modifications. In brief, $10 \mathrm{ml}$ of Buffer P1 was supplemented with $1 \mathrm{ml}$ of zymolyase solution (10 $\mathrm{mg} \mathrm{ml}^{-1}$ Zymolyase $100-\mathrm{T} ; 50 \mathrm{mM}$ Tris-HCl pH 7.5;50\% (v/v) glycerol) and $100 \mu \mathrm{l}$ of $\beta$-mercapthoethanol. The mixture was incubated for $1 \mathrm{~h}$ at $37^{\circ} \mathrm{C}$ before the addition of buffer $\mathrm{P} 2$. The rest of the protocol followed the manufacturer's instructions. DNA preparations were successfully used as templates to generate in vitro transcribed viral RNA even if they contained traces of yeast genomic DNA. In parallel, isolated YACs containing full-length synthetic fragments 5 and 7 , as well as SARS-CoV-2 and SARS-CoV-2-GFP viral genomes, were successfully transformed into $E$. coli TransforMax Epi300 electrocompetent cells (Epicentre) (data not shown).

\section{Stability testing of the YAC containing entire RNA virus genomes in yeast}

The stability of viral genomes maintained as YACs in S. cerevisiae was tested for the clones containing MHV-GFP or MERS-CoV for 1 week. 
A single colony was grown in $20 \mathrm{ml}$ of SD-His liquid medium, $1 \mathrm{ml}$ aliquots were removed and expanded in fresh medium every $12 \mathrm{~h}$. The generation time for each of the clones was estimated to range from 150 to $160 \mathrm{~min}$. After $15-17$ passages, each YAC clone was isolated and subjected to sequencing by MinION (Oxford Nanopore Technologies) to obtain the entire YAC sequence. Individual regions for which MinION sequencing did not reveal a clear sequence were resequenced by Sanger sequencing (Microsynth).

\section{Virus rescue}

The YAC containing viral CDNA was cleaved at the unique restriction site located downstream of the $3^{\prime}$ end poly(A) tail (Extended Data Table 1). In brief, 1-2 $\mu$ g of phenol-chloroform-extracted and ethanol-precipitated restricted DNA was resolved in nuclease-free water and used for in vitro transcription using the T7 RiboMAX Large Scale RNA production system (Promega) with $\mathrm{m} 7 \mathrm{G}\left(5^{\prime}\right) \mathrm{ppp}\left(5^{\prime}\right) \mathrm{G}$ cap provided as described previously ${ }^{2}$. Additionally, a similar protocol was performed on a PCR product of the $N$ gene from corresponding coronaviruses, producing a capped mRNA that encodes the N protein. Then, 1-10 $\mu$ g of in vitro transcribed viral RNA was electroporated together with $2 \mu \mathrm{g}$ of the $N$ gene transcript into BHK-21 cells and/or BHK-21 cells expressing the corresponding coronavirus $\mathrm{N}$ protein. Electroporated cells were co-cultured with susceptible mouse $17 \mathrm{Cl}-1$, Vero B4 and Vero E6 cells to rescue rMHV-GFP (17Cl-1), rMERS-CoV and rMERS-CoV-GFP (Vero B4), and rSARS-CoV-2, rSARS-CoV-2-GFP and synSARS-CoV-2-GFP(VeroE6). Progeny viruses that were collected from the supernatant immediately after electroporation were termed passage 0 viruses and were used to produce stocks for subsequent analysis. Virus-infected cells were monitored, and images were acquired using an EVOS fluorescence microscope equipped with a $10 \times$ air objective. Brightness and contrast were adjusted using FIJI. Figures were assembled using the FigureJ plugin ${ }^{30}$.

All work involving the rescue and characterization of recombinant MERS-CoV, SARS-CoV and SARS-CoV-2 was performed in a biosafety level 3 laboratory at the Institute of Virology and Immunology, Mittelhäusern, Switzerland under appropriate safety measures with respect to personal and environmental protection.

\section{Virus growth kinetics}

In brief, $24 \mathrm{~h}$ before infection with MHV-GFP, L929 cells were seeded in a 24 -well plate at a density of $3.6 \times 10^{5}$ cells per $\mathrm{ml}$. Cells were washed once with PBS and inoculated with viruses (multiplicity of infection $(\mathrm{MOI})=0.1)$. After $2 \mathrm{~h}$, the virus-containing supernatant was removed, and cells were washed three times with PBS and supplied with medium as described above. Cell-culture supernatants were collected at the indicated time points after infection. A similar protocol was used for MERS-CoV and MERS-CoV-GFP using Vero B4 cells $(\mathrm{MOI}=0.01)$, and SARS-CoV-2 using Vero E6 cells $(\mathrm{MOI}=0.01)$. Statistical significance was determined by two-sided unpaired Student's $t$-test without adjustments for multiple comparisons.

\section{Plaque assay and $\mathrm{TCID}_{50}$}

MHV-GFP PFU ml ${ }^{-1}$ was determined by plaque assay in L929 cells as described previously ${ }^{14}$. In brief, 24 h before infection, L929 cells were seeded in a 24 -well plate at a density of $3.6 \times 10^{5}$ cells per $\mathrm{ml}$. Cells were washed with PBS and inoculated with viruses serially diluted in cell-culture medium at 1:10 dilution. Cells were washed with PBS $1 \mathrm{~h}$ after inoculation, and overlaid with $2 \%$ methylcellulose mixed at 1:1 with $2 \times$ DMEM supplemented with $20 \%$ fetal bovine serum, 200 units $\mathrm{ml}^{-1}$ penicillin and $200 \mathrm{\mu g} \mathrm{ml}^{-1}$ streptomycin. After $24 \mathrm{~h}$ of incubation, the overlay was removed and cells were fixed and stained with crystal violet.

The $\mathrm{TCID}_{50}$ assay was performed for MERS-CoV and MERS-CoV-GFP in Vero B4 cells and SARS-CoV-2 and SARS-CoV-2-GFP in Vero E6 cells. In brief, cells were seeded $24 \mathrm{~h}$ before infection in a 96 -well plate at a density of $2 \times 10^{6}$ cells per plate. Viruses were serially diluted at 1:10 dilution from $10^{-1}$ to $10^{-8}$. After $72 \mathrm{~h}$ of incubation, the medium was removed and cells were fixed and stained with crystal violet. The TCID ${ }_{50} \mathrm{ml}^{-1}$ titre was determined using the Spearman-Kaerber method ${ }^{31}$.

The PFU ml ${ }^{-1}$ of SARS-CoV-2 and SARS-CoV-2-GFP was determined by plaque assay using Vero E6 cells in a 6-well format. In brief, $24 \mathrm{~h}$ before infection, Vero E 6 cells were seeded at a density of $2 \times 10^{6}$ cells per plate. At the time of infection, cells were washed with PBS and inoculated with viruses serially diluted in cell-culture medium at 1:10 dilution. Cells were washed with PBS $1 \mathrm{~h}$ after inoculation and overlaid with $2.4 \%$ Avicel mixed at 1:1 with 2× DMEM supplemented with $20 \%$ fetal bovine serum, 200 units $\mathrm{ml}^{-1}$ penicillin and $200 \mathrm{\mu g} \mathrm{ml}^{-1}$ streptomycin. After $48 \mathrm{~h}$ of incubation, the overlay was removed and cells were fixed and stained with crystal violet.

\section{Sequencing and computational analysis}

Full-length sequences of the SARS-CoV-2 and SARS-CoV-2-GFP cDNAs cloned in yeast were confirmed by Sanger sequencing (Microsynth). All other virus genomes cloned in yeast were confirmed using the Nanopore sequencer MinION from Oxford Nanopore Technologies according to standard protocols. The operating software MinKNOW performed data acquisition and real-time base calling, generating data as fast 5 and/or fastq files. Subsequently, the Python command line qcat (Mozilla Public License 2.0., copyright 2018 Oxford Nanopore Technologies, v1.1.0, http://www.github.com/nanoporetech/qcat) was run to demultiplex Nanopore reads from fastq files. Alignment of demultiplexed reads to reference sequences was carried out using the Minimap 2 program ${ }^{32}$, producing a fasta file. Mutations of consensus sequences and regions for which the sequences were not clear were verified by Sanger sequencing (Microsynth).

rSARS-CoV-2 and SARS-CoV-2-GFP RNA was sequenced by next-generation sequencing using poly(A)-purified RNA. In brief, $1 \times 10^{6}$ Vero E6 cells were infected with rSARS-CoV-2 clones 1.1, 2.2, 3.1 and rSARS-CoV-2-GFP clones 4.1, 5.2, 6.2 (all passage 1) at an $\mathrm{MOI}=0.001$. Cellular RNA was prepared using NucleoSpin RNA Plus (Macherey-Nagel) according to the manufacturer's recommendation. The quantity and quality of the extracted RNA was assessed using a Thermo Fisher Scientific Qubit 4.0 fluorometer with the Qubit RNA BR Assay Kit (Thermo Fisher Scientific, Q10211) and an Advanced Analytical Fragment Analyzer System using a Fragment Analyzer RNA Kit (Agilent, DNF-471), respectively. Sequencing libraries were produced using an Illumina TruSeq Stranded mRNA Library Prep kit (Illumina, 20020595) in combination with TruSeq RNA UD Indexes (Illumina, 20022371) according to Illumina's guidelines. Pooled cDNA libraries were paired-end sequenced using an Illumina NovaSeq $6000 \mathrm{~S}$ Prime Reagent Kit (300 cycles; Illumina, 20027465) on an Illumina NovaSeq 6000 instrument, generating an average of 69 million reads per sample. The quality-control assessments, generation of libraries and sequencing run were all performed at the Next Generation Sequencing Platform, University of Bern, Switzerland. For analysis, the adaptor sequences were trimmed using TrimGalore software (v.0.6.5) and reads shorter than 20 nucleotides in length and/or with a Phred score of less than 20 were removed. Paired-end trimmed reads were mapped to the SARS-CoV-2 genome (GenBank accession MT108784; synthetic construct derived from SARS-2 BetaCoV/Wuhan/IVDC-HB-01/2019) using the Spliced Transcripts Alignment to a Reference (STAR) aligner (v.2.7.0a $)^{33}$ with default parameters. Before mapping, STAR was also used to generate a genome index for SARS-CoV-2 with the parameters --genomeSAindexNbases 7 and --sjdbOverhang 149. SAMtools (v.1.10) was used to calculate mapped read depth from the resulting mapped read pairs at each position in the genome and subsequently visualized using a variety of software packages in R. Calculations were performed on UBELIX (http://www.id.unibe.ch/hpc), the HPC cluster at the University of Bern. Sequencing data have been deposited in the Sequence Read Archive (SRA) of the NCBI (http://www.ncbi.nlm.nih.gov/sra).

Apart from MinION and next-generation sequencing data handling, other sequence analyses were performed using Geneious Prime 
v.2019.2.3. Results from virus growth kinetics were analysed and graphically presented using GraphPad Prism v.8.3.0 for Windows. All figures were created with Adobe Illustrator and Biorender.com.

\section{Identification of leader-body junctions of viral mRNAs}

To identify reads that mapped discontinuously to the SARS-CoV-2 genome and determine the location of potential transcription regulatory sites (TRS), we pooled reads that mapped to the viral genome as well as unmapped reads and searched for the sequence TTCTCTAAACGAAC (nucleotides 62-75 of MT108784; leader TRS is indicated in bold). We then filtered for reads that had at least 18 nucleotides $3^{\prime}$ of the aforementioned sequence and evaluated whether these reads were compatible with any of the SARS-CoV-2 mRNA sequences. Reads matching these criteria were used as input for the generation of a consensus sequence for each TRS site and analysed using a combination of SAMtools (v.1.10), R and the Integrative Genomics Viewer (IGV). Mapped read depth was also calculated for the discontinuously mapped reads as explained in the previous section.

\section{5'-RACE}

Recombinant SARS-CoV-2 and SARS-CoV-2-GFP poly(A)-purified RNA used for next-generation sequencing was also used to determine the genome $5^{\prime}$ ends by $5^{\prime}$-RACE. M-MLV reverse transcription (Promega) was performed according to the manufacturer's instructions using the gene-specific primer pWhSF-ORF1a-R18-655 (Supplementary Table 1) and $10 \mathrm{U}$ RNase Inhibitor RNasin plus (Promega) per $25 \mu \mathrm{l}$ reaction volume. Following reverse transcription, $1 \mu \mathrm{l}$ RNase $\mathrm{H}\left(5 \mathrm{U}^{-1}\right.$, New England Biolabs) per $25 \mu \mathrm{l}$ reaction was added, and the mixture was incubated at $37^{\circ} \mathrm{C}$ for $20 \mathrm{~min}$. The cDNA was immediately purified with the High Pure PCR product purification kit (Roche) according to the manufacturer's instructions. A poly(A) tail was added to the cDNA with Terminal Transferase (New England Biolabs) according to the manufacturer's instructions. Subsequently, a PCR reaction with the tailed cDNA was performed with the primer pair pWhSF-ORF1a-R18-655 and TagRACE_dT16 (Supplementary Table 1) using the HotStarTaq Master Mix (QIAGEN) according to the manufacturer's instructions with a touchdown cycling protocol: $95^{\circ} \mathrm{C}$ for $15 \mathrm{~min} ; 15$ cycles of $94^{\circ} \mathrm{C}$ for $30 \mathrm{~s}, 65^{\circ} \mathrm{C}$ touchdown to $50^{\circ} \mathrm{C}$ for $1 \mathrm{~min}, 72^{\circ} \mathrm{C}$ for $1 \mathrm{~min} ; 25$ cycles of $94^{\circ} \mathrm{C}$ for $30 \mathrm{~s}, 50^{\circ} \mathrm{C}$ for $1 \mathrm{~min}, 72^{\circ} \mathrm{C}$ for $1 \mathrm{~min}$. Subsequently, $1 \mu \mathrm{l}$ of this reaction was used for a nested re-amplification with the primer pair pWhSF-5utr-R17-273 and TagRACE (Supplementary Table 1) in a final volume of $50 \mu \mathrm{l}$ following the same cycling protocol as described above. The PCR fragment was purified using the NucleoSpin Gel and PCR Clean-up Kit (Macherey-Nagel) according to the manufacturer's instructions, and the purified PCR fragment was sent to Microsynth for Sanger sequencing with the primer pWhSF-5utr-R17-273 (Supplementary Table 1). Sequencing raw data were assessed using the SeqManTM Il sequence analysis software (DNASTAR).

\section{Remdesivir experiment}

Remdesivir (MedChemExpress) was dissolved in DMSO and stored at $-80^{\circ} \mathrm{C}$ in $20 \mathrm{mM}$ stock aliquots. One day before the experiment, Vero E6 cells were seeded in 24 -well plates at a density of $8 \times 10^{4}$ cells per well. Cells were infected with synSARS-CoV-2-GFP (passage 1 ) at MOI $=0.01$ or mock-infected as control. Innocula were removed at $1 \mathrm{~h}$ after infection, and replaced with medium containing remdesivir $(0.2 \mu \mathrm{M}$ or $2 \mu \mathrm{M})$ or the equivalent amount of DMSO. At $48 \mathrm{~h}$ after infection, cells were washed once with PBS and incubated in fresh PBS. Images were acquired using an EVOS fluorescence microscope equipped with a $10 \times$ air objective. Brightness and contrast were adjusted identically for each condition and their corresponding control using FIJI. Figures were assembled using the FigureJ plugin ${ }^{30}$.

\section{Immunofluorescence assay}

One day before infection, Vero E6 cells were seeded in a 12-well removable chamber glass slide (Ibidi) at a density of $4 \times 10^{4}$ cells per well. Cells were infected with rSARS-CoV-2 clone 3.1 (passage 2) or mock-infected as control. At 6 and $24 \mathrm{~h}$ after infection, cells were washed twice with PBS and fixed with $4 \%(\mathrm{v} / \mathrm{v})$ neutral-buffered formalin. Cells were washed twice with PBS before permeabilization with $0.1 \%$ Triton X-100 and blocking with PBS supplemented with $50 \mathrm{mM} \mathrm{NH}_{4} \mathrm{Cl}, 0.1 \%$ (w/v) saponin and $2 \%(\mathrm{w} / \mathrm{v}) \mathrm{BSA}$ (confocal buffer) for $60 \mathrm{~min}$. Primary antibodies (anti-dsRNA, J2, English and Scientific Consulting, 10010500; and anti-SARS-CoV Nucleocapsid (N), Rockland, 200-401-50) and secondary antibodies (donkey anti-rabbit 594, Jackson ImmunoResearch 711-585152; and donkey anti-mouse 488, Jackson ImmunoResearch 715-545-150) were diluted in confocal buffer. Slides were covered with $0.17-\mathrm{mm}$ thick, high-performance $(1.5 \mathrm{H})$ glass coverslips and mounted using ProLong Diamond Antifade mountant containing 4',6-diamidino-2-phenylindole (DAPI) (Thermo Fisher Scientific). Images were acquired using an EVOS FL Auto 2 Imaging System equipped with a coverslip-correct $40 \times$ air objective. Brightness and contrast were adjusted identically for each condition and their corresponding control using FIJI. Figures were assembled using the FigureJ plugin ${ }^{30}$.

\section{Serum neutralization assay}

One day before the experiment, Vero E6 cells were seeded in a 96-well clear-bottom, black plate at a density of $2 \times 10^{6}$ cells per well. Serum 2 has been described in another study ${ }^{34}$ as patient serum ID7 (convalescent human anti-SARS-CoV-2 serum). Serum 4 has been described previously as patient serum CSS 2 (convalescent human anti-SARS-CoV serum $)^{35}$. Sera 1 and 3 were control sera. In brief, all sera were inactivated for $30 \mathrm{~min}$ at $56^{\circ} \mathrm{C}$ and diluted at 1:10 in OptiMEM. A twofold serial dilution was performed in OptiMEM in a final volume of $50 \mu \mathrm{l}$ in a separate 96 -well plate (dilutions $1: 10$ to $1: 1,280$ ). Then, $50 \mu$ of synSARS-CoV-2-GFP containing $250 \mathrm{TCID}_{50}$ was added to the diluted sera. The serum-virus mixture was incubated at $37^{\circ} \mathrm{C}$ for $60 \mathrm{~min}$, and subsequently added to Vero E6 cells. After $1 \mathrm{~h}$ of incubation, supernatants were removed and replaced with medium as described above. At $48 \mathrm{~h}$ after infection, expression of GFP and cytopathogenic effects were monitored, and images were acquired using an EVOS fluorescence microscope equipped with a $10 \times$ air objective. Brightness and contrast were adjusted identically for each condition and their corresponding control using FIJI. Figures were assembled using the FigureJ plugin ${ }^{30}$.

\section{Ethical statement}

The authors are aware that this work contains aspects of Dual Use Research of Concern (DURC). The benefits were carefully balanced against the risks and the benefits outweigh the risks. Permission to generate and work with recombinant SARS-CoV-2 and SARS-CoV-2-GFP was granted by the Swiss Federal Office of Public Health (A131191/3) with consultation of the Federal Office for Environment, Federal Food Safety and Veterinary Office, and the Swiss Expert Committee for Biosafety.

\section{Reporting summary}

Further information on research design is available in the Nature Research Reporting Summary linked to this paper.

\section{Data availability}

The following genome sequences are available from GenBank: rSARS-CoV-2(MT108784), hRSV/B/Bern/2019(MT107528); MERS-CoV Riyadh-1734-2015 (MN481979). The RNA-sequencing data of rSARS-CoV-2and rSARS-CoV-2-GFPareavailable from the NCBISequence Read Archive (BioProject accession number PRJNA615319; BioSample accessions: SAMN14450686, SAMN14450687, SAMN14450688, SAMN14450689, SAMN14450690 and SAMN14450691). Source data are provided with this paper.

23. V'kovski, P. et al. Determination of host proteins composing the microenvironment of coronavirus replicase complexes by proximity-labeling. eLife 8, e42037 (2019). 
24. Raj, V. S. et al. Dipeptidyl peptidase 4 is a functional receptor for the emerging human coronavirus-EMC. Nature 495, 251-254 (2013).

25. Jonsdottir, H. R. et al. Establishment of primary transgenic human airway epithelial cell cultures to study respiratory virus-host interactions. Viruses 11, 747 (2019).

26. Noskov, V. et al. A genetic system for direct selection of gene-positive clones during recombinational cloning in yeast. Nucleic Acids Res. 30, E8 (2002).

27. Green, M. R. \& Sambrook, J. Molecular Cloning: A Laboratory Manual 4th edn (Cold Spring Harbor Laboratory Press, 2012).

28. Gietz, R. D. \& Schiestl, R. H. High-efficiency yeast transformation using the LiAc/SS carrier DNA/PEG method. Nat. Protoc. 2, 31-34 (2007)

29. Blount, B. A., Driessen, M. R. \& Ellis, T. GC preps: fast and easy extraction of stable yeast genomic DNA. Sci. Rep. 6, 26863 (2016).

30. Mutterer, J. \& Zinck, E. Quick-and-clean article figures with FigureJ. J. Microsc. 252, 89-91 (2013).

31. Hierholzer, J. C. \& Killington, R. A. in Virology Methods Manual (eds Mahy, B. W. J. \& Kangro, H. O.) 25-46 (Academic Press, 1996).

32. Li, H. Minimap2: pairwise alignment for nucleotide sequences. Bioinformatics $\mathbf{3 4}$ 3094-3100 (2018).

33. Dobin, A. et al. STAR: ultrafast universal RNA-seq aligner. Bioinformatics 29, 15-21 (2013).

34. Woelfel, R. et al. Clinical presentation and virological assessment of hospitalized cases of coronavirus disease 2019 in a travel-associated transmission cluster. Preprint at medRxiv https://doi.org/10.1101/2020.03.05.20030502 (2020).

35. Hoffmann, M. et al. SARS-CoV-2 cell entry depends on ACE2 and TMPRSS2 and is blocked by a clinically proven protease inhibitor. Cell 181, 271-280 (2020).

36. Chan, J. F. et al. Genomic characterization of the 2019 novel human-pathogenic coronavirus isolated from a patient with atypical pneumonia after visiting Wuhan. Emerg. Microbes Infect. 9, 221-236 (2020).
Acknowledgements This work was supported by the European Commission (Marie Skłodowska-Curie Innovative Training Network "HONOURS"; grant agreement no. 721367), the Swiss National Science Foundation (SNF; grants CRSII3_160780 and 310030_173085), the German Research Council (DFG; grants SFB-TR84 (TRR 84/3, A07) and DR 772/7-2), the Federal Ministry of Education and Research (BMBF; grant RAPID, 01KI1723A) and by core funds of the University of Bern. We thank S. Vashee for the provision of the TAR vectors and for discussions related to his herpesvirus work; J. Peters Zocher for her advice in generating the figures; F. Suter-Riniker and P. Bittel for providing clinical samples; staff from the Next Generation Sequencing platform (University of Bern); and M. Schweizer, P. Plattet, M. Gerber, M. Friesland, M. Alves, N. Vielle, B. Zumkehr, M. Brügger and D. Brechbühl for reagents, technical advice and helpful discussions. This study is dedicated to S. Kunz.

Author contributions V.T. and J.J. conceived the study. T.T.N.T., N.E. and F.L. performed most of the experiments. P.V., H.S., K.S., J.P., S. Steiner, M.H., A.K., M.G., L.L., L.H., M.W., S.P., D.H., V.C., S.C.-P., S. Schröder, D.M., D.N., V.M.C., M.A.M., C.D. and R.D. did experimental work and/or provided essential experimental systems and reagents. T.T.N.T., N.E., F.L., H.S., J.K. and R.D. performed sequencing analyses, including computational analyses. V.T., J.J., T.T.N.T., F.L. and N.E. wrote the manuscript and made the figures. All authors read and approved the final manuscript.

Competing interests The authors declare no competing interests.

\section{Additional information}

Supplementary information is available for this paper at https://doi.org/10.1038/s41586-0202294-9.

Correspondence and requests for materials should be addressed to J.J. or V.T.

Peer review information Nature thanks Luis Enjuanes, Stanley Perlman and Timothy Patrick Sheahan for their contribution to the peer review of this work.

Reprints and permissions information is available at http://www.nature.com/reprints. 


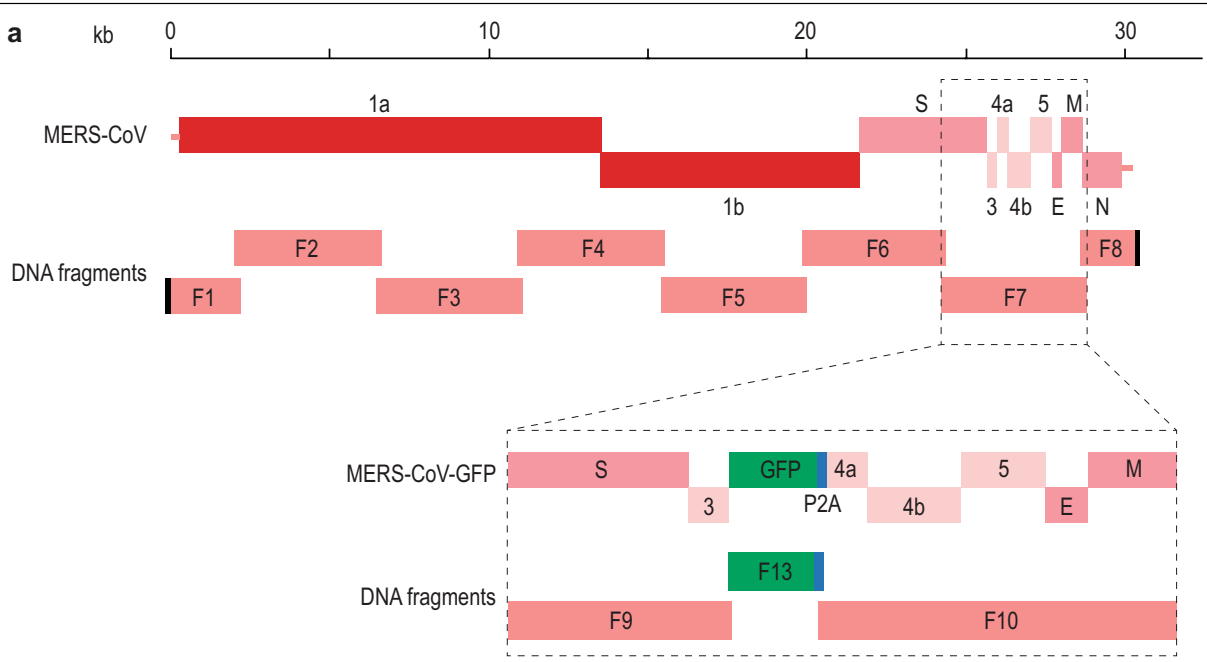

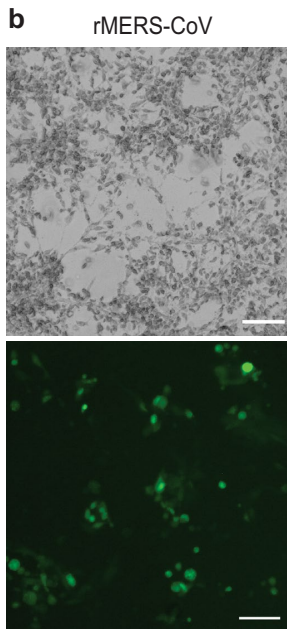

rMERS-CoV-GFP

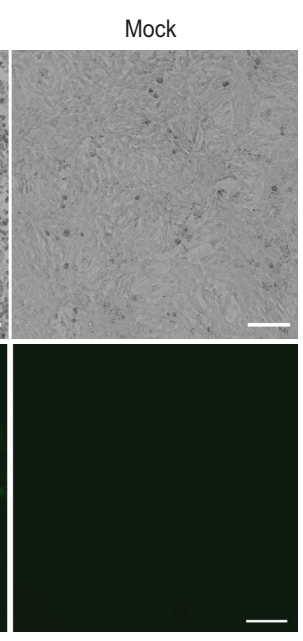

Mock

Extended Data Fig. 1 | Generation of viral cDNA clones and recovery of recombinant MERS-CoV and MERS-CoV-GFP. a, Schematic representation of the genome organization of MERS-CoV (top) and MERS-CoV-GFP (bottom) with 8 and 10 viral subgenomic overlapping fragments used for TAR cloning, respectively. b, Rescue of recombinant MERS-CoV and MERS-CoV-GFP. After the delivery of viral RNAs into BHK-21 cells using electroporation, the cells were co-cultured with Vero B4 cells, and supernatants containing recombinant viruses that were produced were used to infect new Vero B4 cells. Infected cells were visualized by bright-field microscopy for rMERS-CoV (top; 5 days after infection), and by fluorescence microscopy for GFP expression of rMERS-CoV-GFP (bottom, 3 days after infection). Mock, Vero B4 cells inoculated with the supernatant of BHK-21 cells that were electroporated

C

\begin{tabular}{lllll|}
$*$ & $n s$ & $n s$ & $n s$ & $-0-1$ \\
$*$ & $n s$ & $n s$ & $*$ & $-\Delta$ \\
ns & $n s$ & $n s$ & $*$ & $-\triangle$ \\
\hline
\end{tabular}

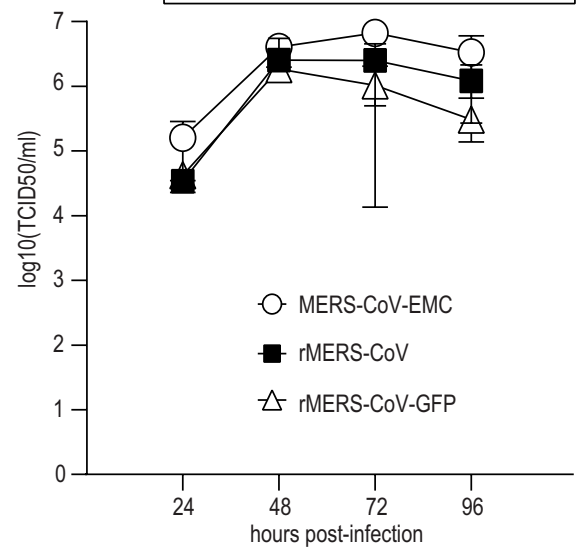

without viral RNAs. Images are representative of two independent experiments. Scale bars, $100 \mu \mathrm{m}$. c, Replication kinetics of MERS-CoV-EMC, rMERS-CoV and rMERS-CoV-GFP. Vero B4 cells were infected $(\mathrm{MOI}=0.01$ ). Cell-culture supernatants were collected at the indicated time points after infection and titrated by $\mathrm{TCID}_{50}$ assay. Data are the mean \pm s.d. of three independent biological replicates. Statistical significance was determined by two-sided unpaired Student's $t$-test without adjustments for multiple comparisons. $P$ values (from left to right): top, ${ }^{*} P=0.0332 ; \mathrm{ns}, P=0.3294$; ns, $P=0.2003$; ns, $P=0.0966$; middle ${ }^{*} P=0.0457$; ns, $P=0.1233$; $\mathrm{ns}, P=0.0838$; ${ }^{*} P=0.0199$; bottom, ns, $P=0.3240$; ns, $P=0.6641$; ns, $P=0.1376$; ${ }^{*} P=0.0427$. TCID50/ml, 50\% tissue culture infectious dose per $\mathrm{ml}$. 
a

Base

SARS-CoV-2 auuaaggguuuauaccuucccagguaacaaaccaaccaacuuucgaucucuuguagaucuguu

Clone 6.2

SARS-COV

Clone 2.2

Clone 5.2

Bat SARS-related CoVs

Clone 1.1

Clone 4.1

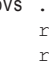

(1,

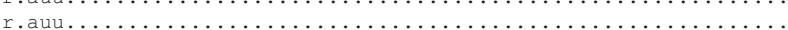

Base

SARS-CoV-2

Clone 3.1

Clone 6.2

SARS-COV

Clone 2.2

Clone 5.2

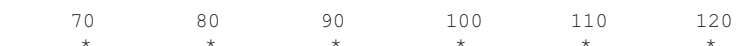

cucuaaacgaacuuuaaaaucuguguggcugucacucggcugcaugcuuagugcacucacg

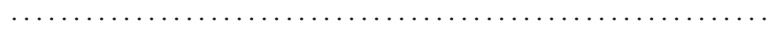

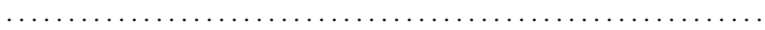

$\ldots \ldots \ldots \ldots \ldots \ldots \ldots \ldots \ldots, \ldots \ldots, \ldots \ldots \ldots \ldots$. $\ldots \ldots \ldots$. $\ldots$.

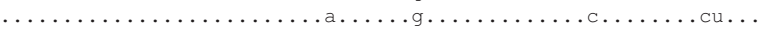

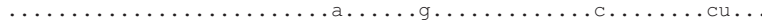

Bat SARS-related CoVs

Clone 1.1

Clone 4.1

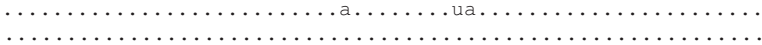

…

b

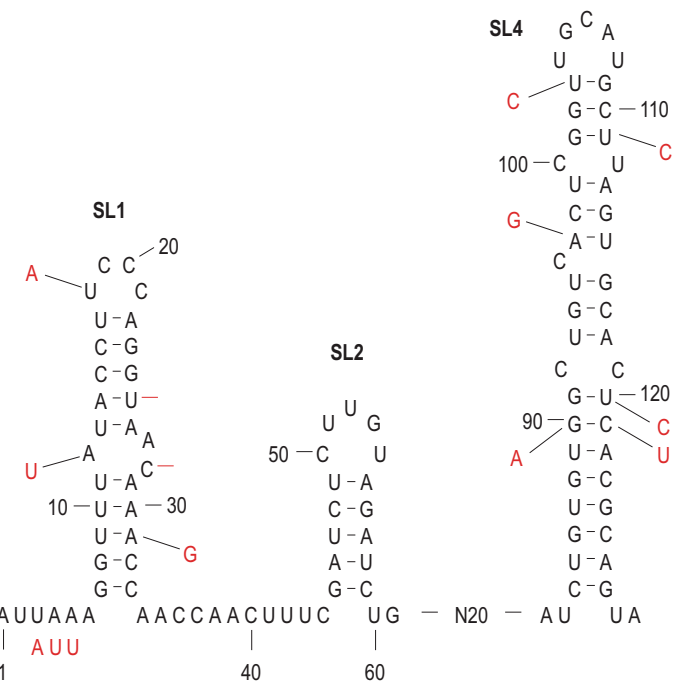

5' UTR nt 1 - 124: SARS-CoV-2

Constructs 3 and 6

C

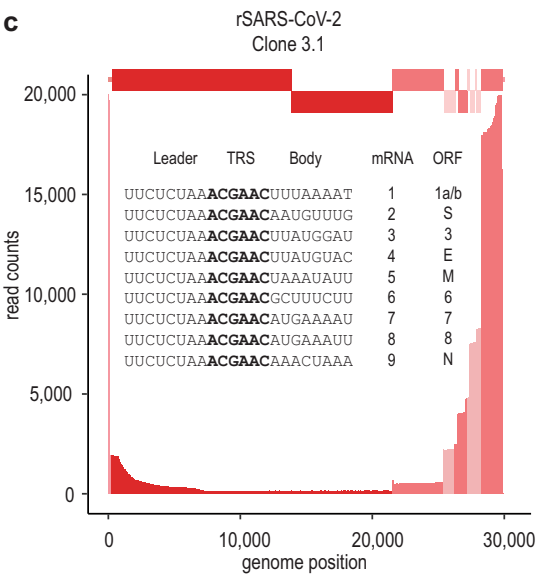

f

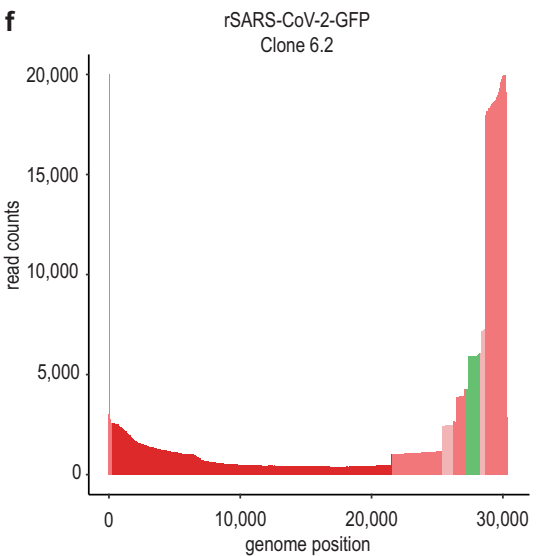

d

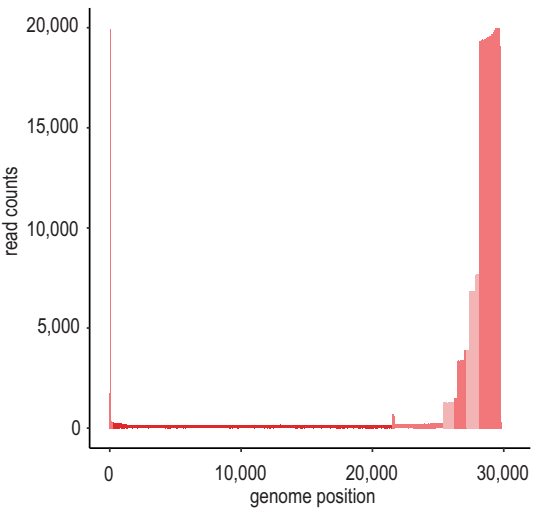

g

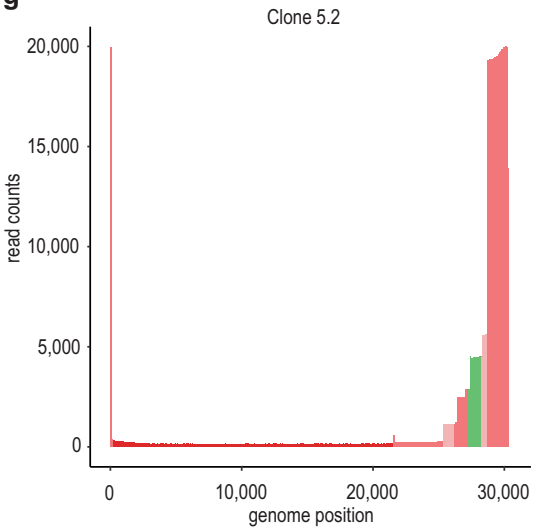

5' UTR nt 1 - 124: SARS-CoV-2 modified by 3 nt Constructs 1 and 4

e $\quad$ rSARS-CoV-2

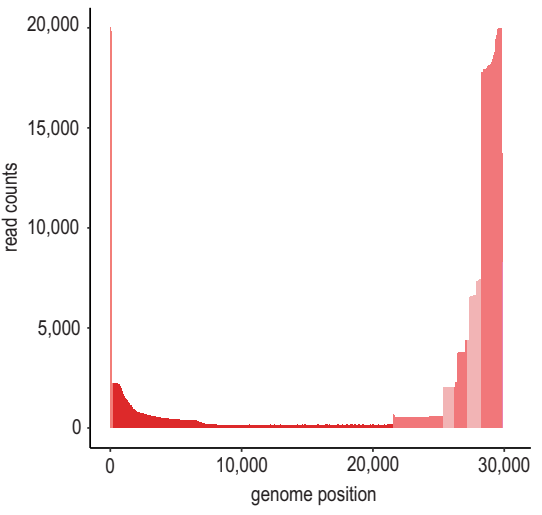

h $\quad$ ISARS-COV-2-GFP

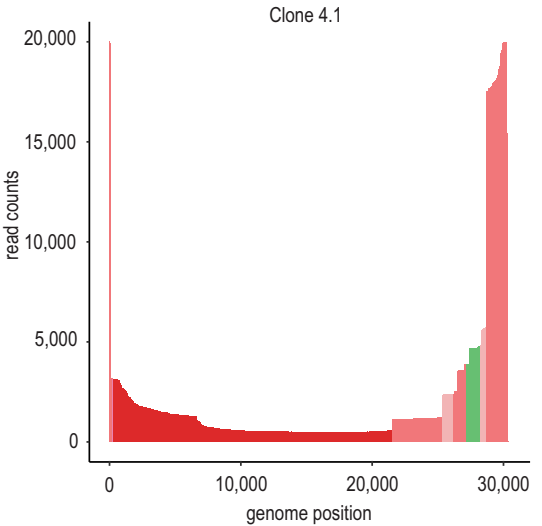

Extended Data Fig. 2 |See next page for caption. 


\section{Article}

Extended Data Fig. 2 | Sequence analyses of the 5' UTR of the SARS-CoV-2 genome. a, Sequence analysis using 5'RACE. Results from 5'RACE of rescued rSARS-CoV-2 and rSARS-CoV-2-GFP clones are shown as a sequence comparison of the first 124 nucleotides of the $5^{\prime}$ UTR region of the SARS-CoV-2 genome (top; MN996528.1) used to generate clones 3.1 and 6.2; the SARS-CoV Frankfurt-1 isolate (middle; AY291315) used to generate clones 2.2 and 5.2 and bat SARSrelated CoVs (bottom; ZXC21 and ZC45) used to generated clones 1.1 and 4.1. A 5'-RACE analysis has been performed from viral RNA for all clones and the sequence has been confirmed.b, Representation of predicted RNA stem-loop (SL) secondary structures within the 5' UTR of SARS-CoV-2. The secondary structures of SARS-CoV-2 RNA were manually adjusted based on previously published RNA structure predictions ${ }^{36}$. Black letters and numbers represents the SARS-CoV-2 5'-terminal sequence. Red letters depict nucleotides that are different within the SARS-CoV 5'-terminal sequence (the '-' indicates a nucleotide deletion in SARS-CoV compared with SARS-CoV-2). N20 indicates 20 nucleotides. $\mathbf{c}-\mathbf{h}$, RNA-sequencing analysis of rSARS-CoV-2 clones 3.1 (c), 2.2 (d), 1.1 (e) and rSARS-CoV-2-GFP clones 6.2 (f), 5.2 (g), 4.1(h). The sequence read coverage of the SARS-CoV-2 and SARS-CoV-2-GFP genomes is shown as read counts plotted according to the genome positions. The sequence read coverage is colour-coded according to the viral ORFs (red, ORF1a/b; dark pink, structural genes; light pink, accessory genes; green, GFP) to illustrate the characteristic pattern of the coronavirus transcription gradient of genomic and subgenomic viral RNAs. Leader-body junctions of viral RNAs were determined for SARS-CoV-2 clone 3.1 and are depicted in c. RNAs used for 5'-RACE (a) and RNA-sequencing analyses $(\mathbf{c}-\mathbf{h})$ were prepared from virusinfected Vero E6 cells ( $\mathrm{MOI}=0.001 ; 48 \mathrm{~h}$ after infection). The TRS is highlighted in bold. 


\begin{tabular}{|c|c|c|c|c|c|}
\hline 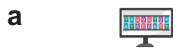 & Design & & & (0) & ning \\
\hline \multirow{4}{*}{ rSARS-CoV-2 } & \multirow{4}{*}{ gese } & & $\begin{array}{l}1 \\
1 \\
1\end{array}$ & $\begin{array}{l}\text { Positive out of } \\
10 \text { screened clones }\end{array}$ & $\begin{array}{c}\text { YACs for } \\
\text { subsequent rescue }\end{array}$ \\
\hline & & Construct 1 & i & 10 & Clones 1.1 and 1.2 \\
\hline & & Construct 2 & i & 10 & Clones 2.1 and 2.2 \\
\hline & & Construct 3 & $\begin{array}{l}1 \\
1 \\
1\end{array}$ & 9 & Clones 3.1 and 3.2 \\
\hline \multirow{3}{*}{ rSARS-CoV-2-GFP } & \multirow{3}{*}{ 8se } & Construct 4 & i & 10 & Clones 4.1 and 4.2 \\
\hline & & Construct 5 & i & 10 & Clones 5.1 and 5.2 \\
\hline & & Construct 6 & 1 & 9 & Clones 6.1 and 6.2 \\
\hline
\end{tabular}

b Virus rescue and characterisation

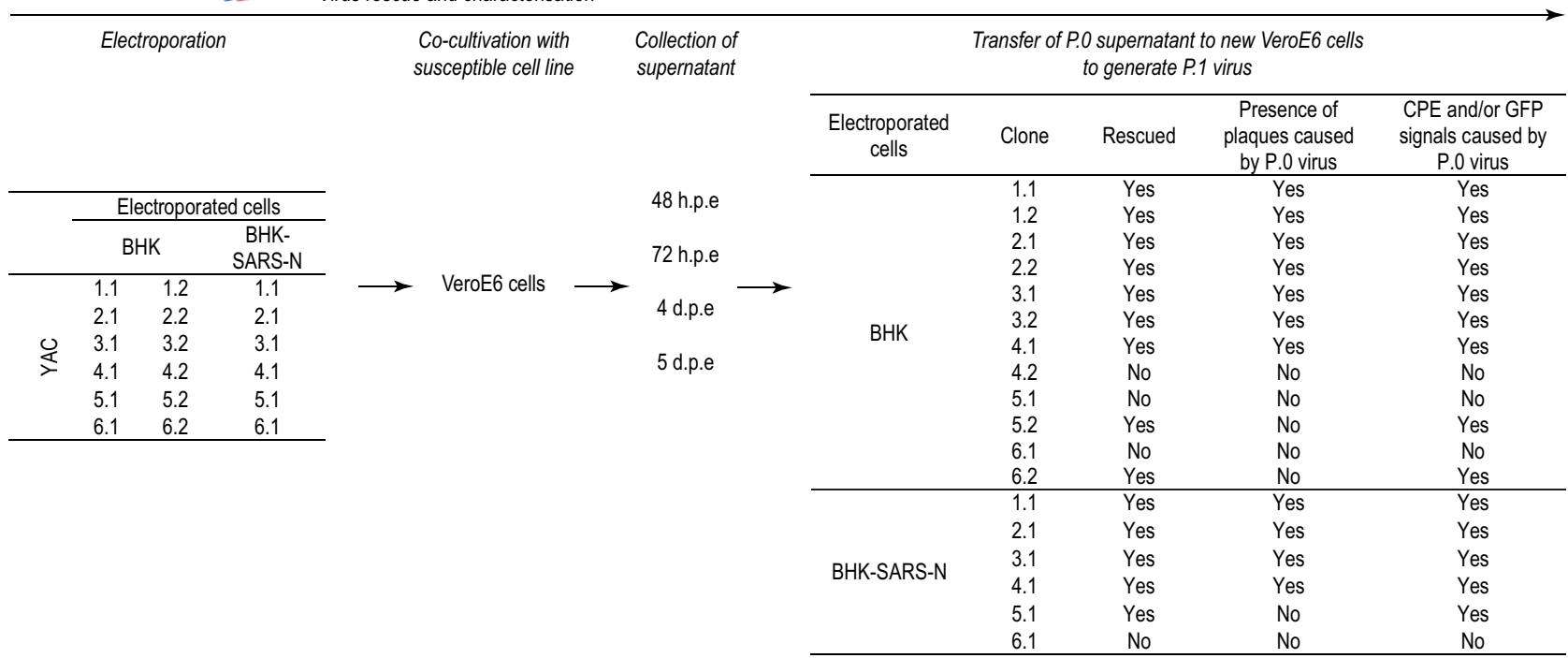

Extended Data Fig. 3 | Workflow for the reconstruction and rescue of rSARS-CoV-2 and rSARS-CoV-2-GFP. a, Overview of the constructs and clones. Six constructs were initially designed on the basis of three different $5^{\prime}$-UTR regions. These regions comprised a modified sequence of the $5^{\prime}-\mathrm{UTR}$ region of SARS-CoV-2 (5'-ATAUUAGG) in which nucleotides 3-5 (UAA) of SARS-CoV-2 were changed to AUU to match nucleotides 3-5 of bat SARS-related CoV (constructs 1 and 4); a SARS-CoV-25'-terminus in which the first 124 nucleotides were changed to the corresponding 5 '-terminal sequence of SARS-CoV (constructs 2 and 5); and the reported sequence of the SARS-CoV-2 (MN996528.1) (constructs 3 and 6). After transformation in yeast, ten colonies were randomly picked for each of the six constructs and all of the junctions bridging the overlapping fragments were verified by multiplex PCR. For each construct, two clones ( $x .1$ and $x .2)$ were randomly selected and YAC DNAs were isolated (12 clones in total). b, Rescue of rSARS-CoV-2 and rSARS-CoV-2-GFP clones. RNAs were generated from YAC DNAs by in vitro transcription and electroporated together with an mRNA encoding the SARS-CoV-2 N protein either into BHK-21 cells (12 clones) or BHK-SARS-N cells (cells expressing the SARS-CoV N protein) (6 clones). Electroporated cells were then co-cultured with susceptible Vero E6 cells to rescue the recombinant viruses. Passage 0 (P.0) supernatants were collected at different time points after electroporation (from 2 to 5 days after electroporation) and transferred to Vero E6 cells to generate passage 1(P.1) virus stocks, and in parallel to demonstrate the presence of infectious virus in plaque assays (for virus clones that do not encode GFP) or fluorescence microscopy (for GFP-encoding virus clones). h.p.e, hours post-electroporation; d.p.e, days post-electroporation; CPE, cytopathogenic effects. 


\section{Article}

a
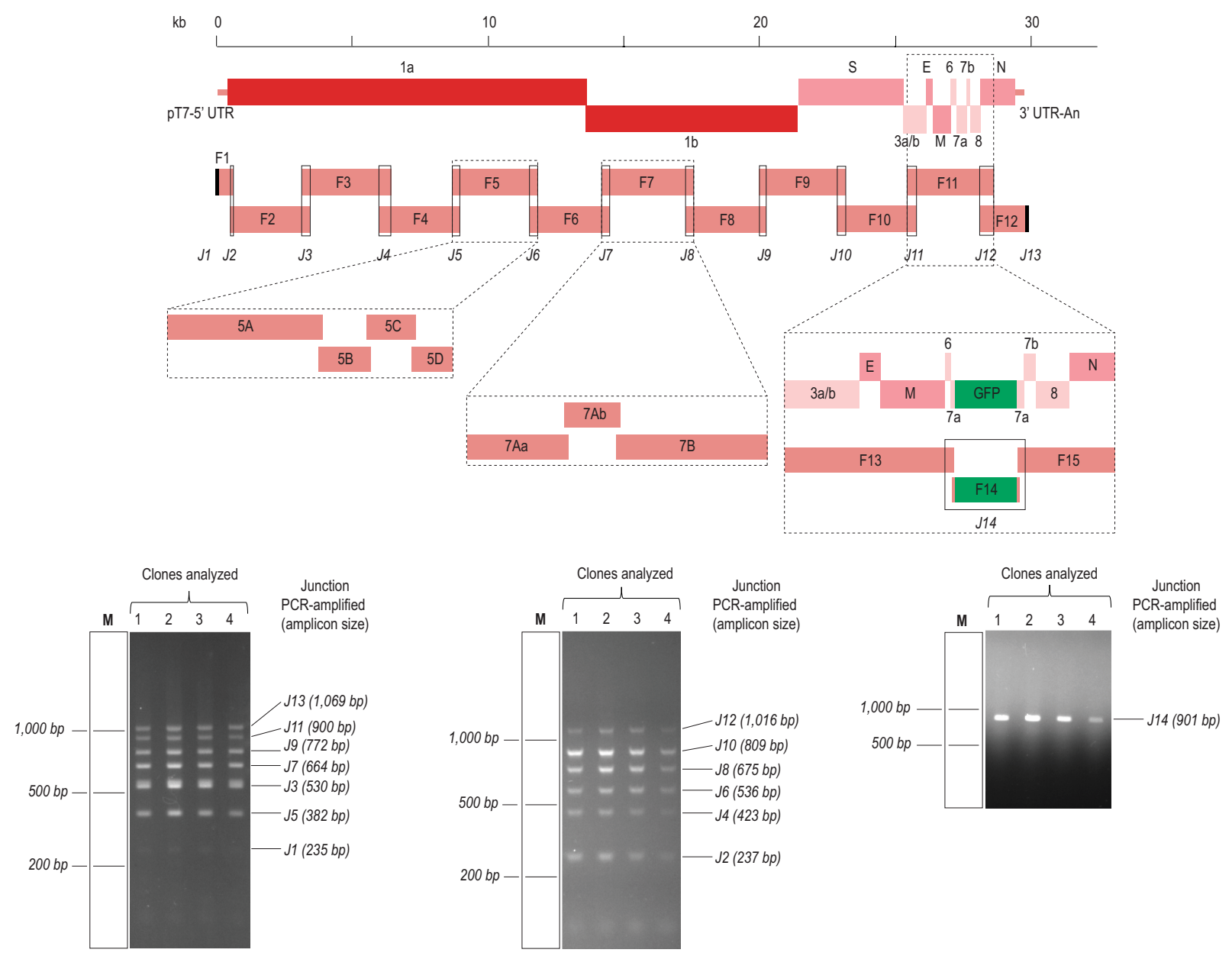

b
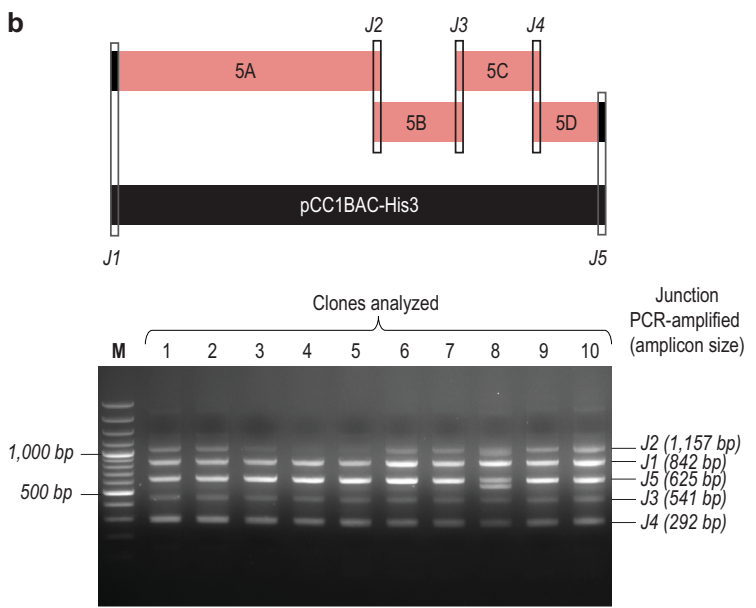

C

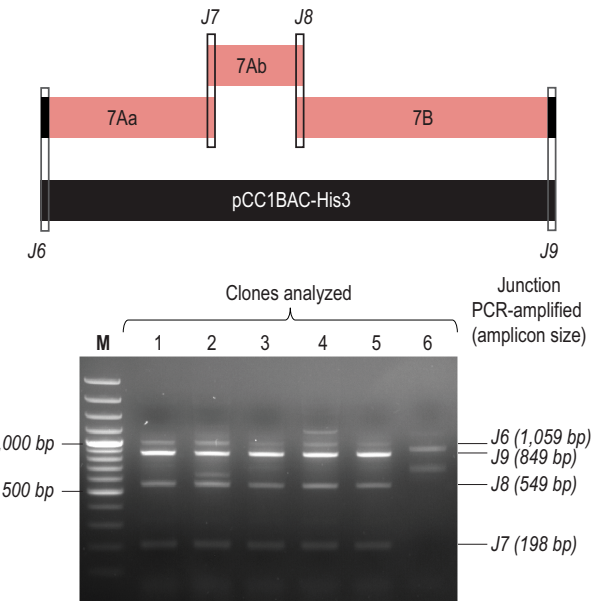

Extended Data Fig. 4 |See next page for caption. 
Extended Data Fig. 4 | Reconstruction of synSARS-CoV-2-GFP and TAR cloning of full-length synthetic fragments 5 and 7 in yeast. $a$, Genome organization of the synSARS-CoV-2-GFP and 19 viral fragments used for TAR cloning (F1-F10; F12-F15). Fragments 5 and 7 were split in four (5A-5D) and three (7Aa, 7Ab, 7B) DNA parts, respectively. Viral ORFs, the ORF for GFP and sequence elements at the $5^{\prime}$ UTR and $3^{\prime}$ UTR are indicated. Primers used to generate the fragments are listed in Supplementary Table 1.J2-J12 and J14 represent the junctions, that is, overlapping regions, between the subgenomic fragments. J1 and J13 represent junctions with the TAR vector. Gel images show the results of two multiplex PCRs designed to confirm the presence of correctly recombined junctions. Multiplex PCR using set 1 primers (left) detects junctions $\mathrm{J1}, \mathrm{J} 3, \mathrm{~J} 5, \mathrm{~J} 7, \mathrm{~J} 9, \mathrm{~J} 11$ and $\mathrm{J} 13$, and multiplex PCR using set 2 primers (middle) detects junctions $\mathrm{J} 2, \mathrm{J4}, \mathrm{J6}, \mathrm{J} 8, \mathrm{~J} 10$ and $\mathrm{J} 12$. The presence of the GFP gene inserted in fragment 14 was confirmed (right). PCR-product sizes are depicted and confirm the proper assembly of the synSARS-CoV-2 full-length genome in all four YAC clones analysed. $\mathbf{b}$, TAR cloning of the full-length synthetic fragment 5 in yeast. Four overlapping synthetic DNA fragments (5A-5D) provided by Genscript were reconstructed as a YAC after transformation in yeast. Correct reassembly was confirmed by multiplex PCR over junctions J1-J5 for 9 out of the10 clones screened (clone 8 was considered incorrect).c, TAR cloning of the full-length synthetic fragment 7 in yeast. Fulllength fragment 7 was assembled by TAR cloning using 3 synthetic dsDNA parts (7Aa, 7Ab and 7B) provided by Genscript. Correct reassembly was confirmed by multiplex PCR over junctions J6-J9 for 5 out of 6 clones (clone 6 is considered incorrect). Cloning experiments shown in a-c have been performed once. pT7, T7 RNA polymerase promoter; An, poly(A) tail; M, GeneRuler 100-bp plus DNA marker (ThermoScientific). 


\section{Article}

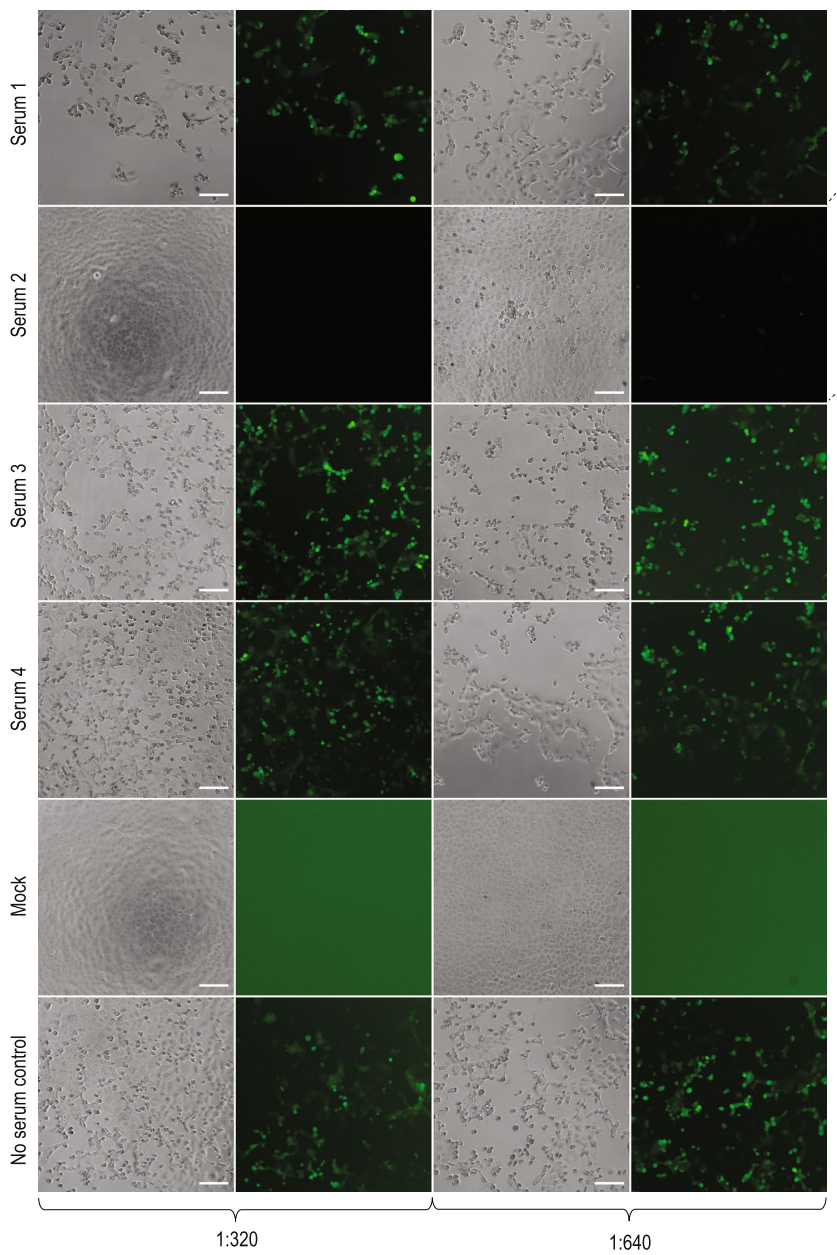

d

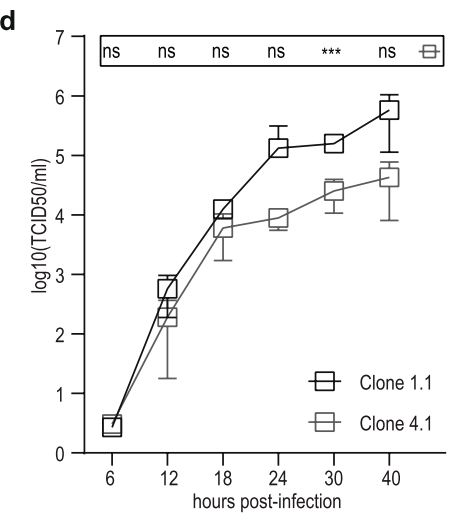

e

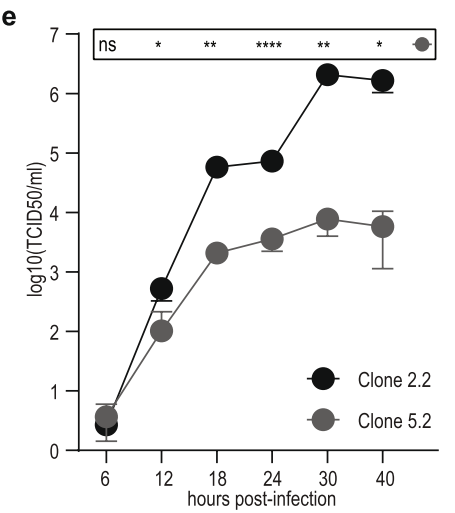

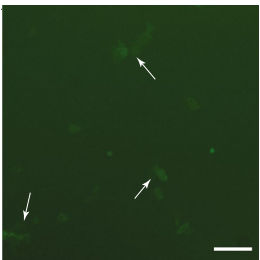

b
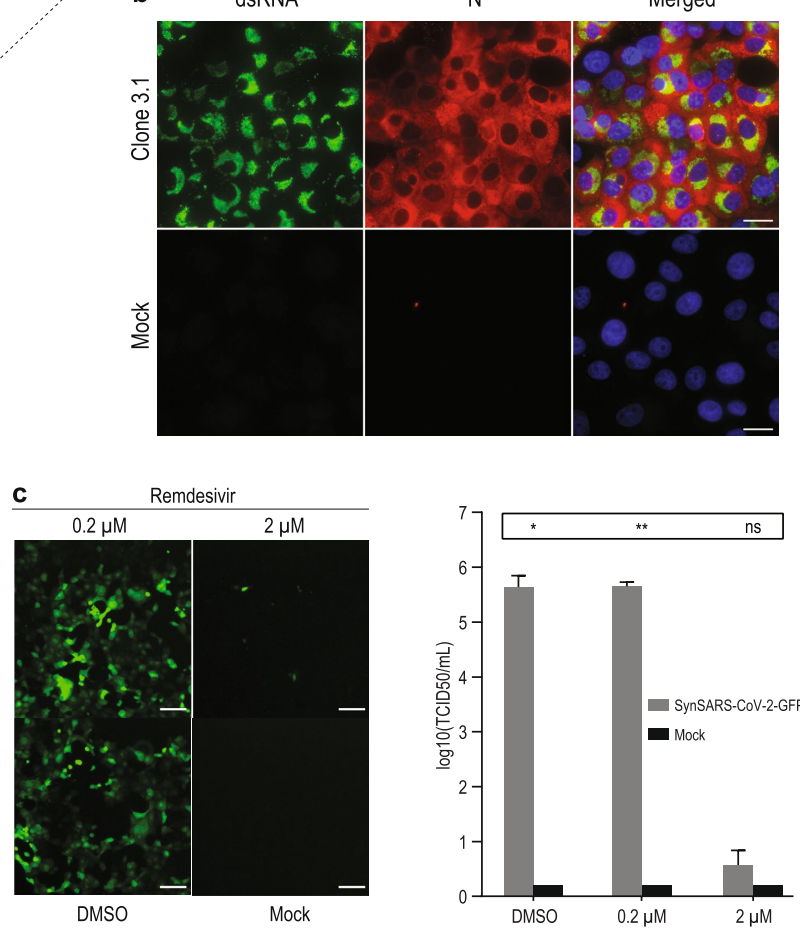

f

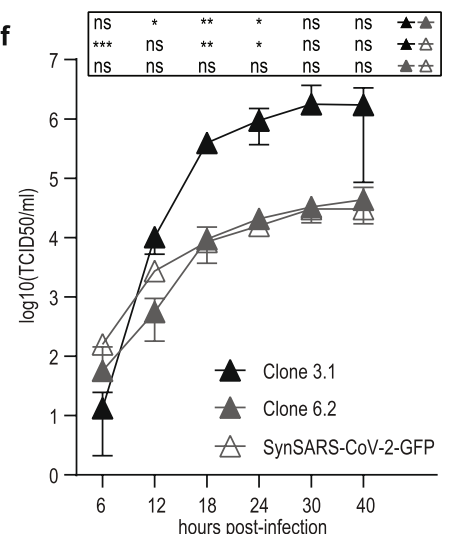

Extended Data Fig. 5 | See next page for caption. 
Extended Data Fig. 5 | Characterization of rSARS-CoV-2, rSARS-CoV-2-GFP and synSARS-CoV-2-GFP. a, Serum neutralization assay showing the use of synSARS-CoV-2-GFP. Inactivated sera were serially diluted and incubated with synSARS-CoV-2-GFP $\left(250 \mathrm{TCID}_{50}\right)$ for $1 \mathrm{~h}$ before infection of Vero E6 cells. Results at $48 \mathrm{~h}$ after infection show that at the virus dilution of 1:320 in serum, virus infection was not observed in serum 2 (convalescent human anti-SARSCoV-2 serum). At the following dilution (1:640), GFP expression was detected, but cytopathogenic effects were not yet detected. Sera 1 and 3, control sera; serum 4 , convalescent human anti-SARS-CoV serum ${ }^{35}$. The serum neutralization experiment was performed twice with similar results. Mock, uninfected cells; no serum control, infected cells with no serum added. b, Immunofluorescence assay showing viral protein synthesis. Vero E6 cells were infected with rSARS-CoV-2 clone 3.1 (passage 2) $(\mathrm{MOI}=0.01)$. Mock, uninfected cells. At $48 \mathrm{~h}$ after infection, cells were fixed and prepared for immunofluorescence staining with primary antibodies directed against dsRNA and SARS-CoV nucleocapsid (N). Green, dsRNA; red, viral N protein; blue, DAPI. The experiment was performed twice with different Vero E6 cells (passages 10 and 11) with similar results. c, Remdesivir treatment showing the use of synSARS-CoV-2-GFP. Vero E6 cells were infected with synSARS-CoV-2-GFP $(\mathrm{MOI}=0.01)$ and treated with $0.2 \mu \mathrm{M}, 2 \mu \mathrm{M}$ or without remdesivir. DMSO was used as treatment in cells that were not incubated with remdesivir. Mock, uninfected cells. At $48 \mathrm{~h}$ after infection, cells were analysed by fluorescence microscopy to detect GFP expression (left) and cell-culture supernatants were collected and titrated by $\mathrm{TCID}_{50}$ assay (right). Titration data represent the mean \pm s.d. of three independent biological replicates $(n=3)$. Statistical significance was determined for synSARS-CoV-2 compared with mock by twosided unpaired Student's $t$-test without adjustment for multiple comparisons. $P$ values (from left to right): ${ }^{*} P=0.0460 ;{ }^{* *} P=0.0010 ; \mathrm{ns}, P=0.2972$. Scale bars, $100 \mu \mathrm{m}(\mathbf{a}-\mathbf{c}) . \mathbf{d}-\mathbf{f}$, Comparison of replication kinetics of rSARS-CoV-2, rSARSCoV-2-GFP and synSARS-CoV-2-GFP. Data are from the same experiment as shown in Fig. $3 \mathrm{c}$ but each panel now shows the direct comparison of rSARSCoV-2, rSARS-CoV-2-GFP and synSARS-CoV-2-GFP clones that have the same 5' terminus: clones 1.1 and 4.1 (5'-AUAUUAGG) (d), clones 2.2 and 5.2 (124 5'-terminal nucleotides of SARS-CoV) (e) and clones 3.1, 6.2 and synSARS-CoV2-GFP (authentic SARS-CoV-2 sequence (5'-AUUAAAGG) according to MN996528.1) (f). Vero E6 cells were infected $(\mathrm{MOI}=0.01)$ and supernatants were collected at indicated time points after infection and titrated by $\mathrm{TCID}_{50}$ assay. Data represent the mean \pm s.d. of three independent biological replicates. Statistical significance was determined by two-sided unpaired Student's $t$-test without adjustments for multiple comparisons. d, $P$ values (from left to right): ns, $P=0.5182 ; \mathrm{ns}, P=0.1920 ; \mathrm{ns}, P=0.0993 ; \mathrm{ns}, P=0.3001$; ${ }^{* * *} P=9.1 \times 10^{-5}$.e, $P$ values (from left to right): ns, $P=0.4978 ;{ }^{*} P=0.0324$; ${ }^{* *} P=0.0020{ }^{* * * *} P<10^{-6} ;{ }^{* *} P=0.0011 ;{ }^{*} P=0.1000 . \mathrm{f}, P$ values (from left to right): top, ns, $P=0.4427 ;{ }^{*} P=0.02474 ;{ }^{* *} P=0.0019 ;{ }^{*} P=0.0490 ; \mathrm{ns}, P=0.1867$; ns, $P=0.1502 ;$ middle, ${ }^{* * *} P=2.4 \times 10^{-5} ; \mathrm{ns}, P=0.1109 ;{ }^{* *} P=0.0019 ;{ }^{*} P=0.4825$; ns, $P=0.1862$; ns, $P=0.1478$; last, ns, $P=0.1161$; ns, $P=0.4026$; ns, $P=0.8700$; ns, $P=0.1161 ; \mathrm{ns}, P=0.8626 ; \mathrm{ns}, P=0.4502$. 


\section{Article}

Extended Data Table 1 | List of restriction enzymes used

to linearize the YAC DNA for each virus before in vitro

transcription

\begin{tabular}{ll}
\hline Virus & $\begin{array}{l}\text { Restriction } \\
\text { enzyme }\end{array}$ \\
\hline MHV-GFP & Pacl \\
MERS-CoV & Mlul \\
MERS-CoV-GFP & Mlul \\
HCoV-229E & Eagl \\
HCoV-HKU1 & Eagl \\
MERS-CoV-Riyadh-1734-2015 & Mlul \\
ZIKA virus & Pacl \\
Human RSV-B & $n . a$ \\
SARS-CoV-2 & Eagl \\
SARS-CoV-2-GFP & Eagl \\
\hline
\end{tabular}

n.a., not applicable. 
Extended Data Table 2 | Stability of the MHV-GFP and MERS-CoV YAC clones in yeast

\begin{tabular}{|c|c|c|c|c|c|c|c|c|}
\hline & \multicolumn{8}{|c|}{ YAC clones } \\
\hline & \multicolumn{4}{|c|}{ MHV-GFP } & \multicolumn{4}{|c|}{ MERS-CoV } \\
\hline & \multicolumn{2}{|c|}{ Clone 1} & \multicolumn{2}{|c|}{ Clone 2} & \multicolumn{2}{|c|}{ Clone 1} & \multicolumn{2}{|c|}{ Clone 2} \\
\hline & Passage 1 & Passage 17 & Passage 1 & Passage 17 & Passage 1 & Passage 15 & Passage 1 & Passage 15 \\
\hline $\begin{array}{l}\text { Non-native viral } \\
\text { sequence(s) }\end{array}$ & none & none & none & none & none & none & none & none \\
\hline $\begin{array}{l}\text { Mutation(s) due to } \\
\text { serial passaging }\end{array}$ & none & none & none & none & none & none & none & none \\
\hline $\begin{array}{l}\text { Mutation(s) due to } \\
\text { RT-PCR }\end{array}$ & 1 & 1 & none & none & none & none & none & none \\
\hline
\end{tabular}




\section{Article}

Extended Data Table 3 | Details of the synthetic HCoV-HKU1, SARS-CoV-2, MERS-CoV Riyadh-1734-2015 and ZIKA virus fragments

\begin{tabular}{|c|c|c|c|c|c|c|c|c|}
\hline \multirow{2}{*}{ Fragment } & \multicolumn{2}{|c|}{$\begin{array}{l}\text { Genome position } \\
\text { (5' UTR to 3' UTR) }\end{array}$} & \multirow{2}{*}{$\begin{array}{l}\text { Fragment size } \\
\text { (bp) }\end{array}$} & \multirow{2}{*}{$\begin{array}{l}\text { Cloning } \\
\text { vector }\end{array}$} & \multirow{2}{*}{$\begin{array}{l}\text { Antibiotic } \\
\text { selection }\end{array}$} & \multicolumn{3}{|c|}{ Restriction sites } \\
\hline & 5'-end & 3'-end & & & & 5'-end & 3'-end & $\begin{array}{c}\text { Vector } \\
\text { digestion }\end{array}$ \\
\hline \multicolumn{9}{|l|}{ HCoV-HKU1 } \\
\hline Fragment 1 & 1 & 2399 & 2506 & pUC57 & $A m p^{R}$ & & & \\
\hline Fragment 2 & 8526 & 10920 & 2395 & pUC57 & $A m p^{R}$ & & & \\
\hline Fragment 3 & 19107 & 21530 & 2424 & pUC57 & $A m p^{R}$ & & & \\
\hline Fragment 4 & 27484 & 29925 & 2646 & pUC57 & $A m p^{R}$ & & & \\
\hline \multicolumn{9}{|l|}{ SARS-CoV-2 } \\
\hline Fragment 1.1 & 1 & 483 & 579 & pUC57 & $A m p^{R}$ & Smal & Smal & \\
\hline Fragment 1.2 & 1 & 483 & 579 & pUC57 & $A m p^{R}$ & Smal & Smal & \\
\hline Fragment 1.3 & 1 & 483 & 579 & pUC57 & $A m p^{R}$ & Smal & Smal & \\
\hline Fragment 2 & 377 & 3325 & 2949 & pUC57 & $A m p^{R}$ & Smal & Smal & Pvul \\
\hline Fragment 3 & 3012 & 6315 & 3304 & pUC57 & $A m p^{R}$ & EcoRV & EcoRV & Pvul \\
\hline Fragment 4 & 6003 & 8994 & 2992 & pUC57mini & $A m p^{R}$ & EcoRV & Ndel & \\
\hline Fragment 5 & 8718 & 11966 & 3249 & pCC1-His3 & $\mathrm{Chlo}^{\mathrm{R}}$ & Smal & Smal & \\
\hline Fragment $5 A$ & 8718 & 10475 & 1757 & pUC19 & $A m p^{R}$ & Smal & & \\
\hline Fragment $5 B$ & 10446 & 11023 & 573 & pUC19 & $A m p^{R}$ & & & \\
\hline Fragment 5C & 10994 & 11540 & 573 & pUC19 & $A m p^{R}$ & & & \\
\hline Fragment $5 D$ & 11511 & 11966 & 456 & pUC19 & $A m p R$ & & Smal & \\
\hline Fragment 6 & 11664 & 14605 & 2942 & pUC57mini & $A m p^{R}$ & Smal & Smal & \\
\hline Fragment 7 & 14311 & 17698 & 3388 & pCC1-His3 & $\mathrm{Chlo}^{\mathrm{R}}$ & Smal & Smal & \\
\hline Fragment 7Aa & 14311 & 15445 & 1134 & & & Smal & & \\
\hline Fragment $7 A b$ & 15416 & 16029 & 614 & & & & & \\
\hline Fragment $7 B$ & 16000 & 17698 & 1699 & pUC57 & $A m p{ }^{R}$ & & Smal & \\
\hline Fragment 8 & 17399 & 20358 & 2960 & pUC57mini & $A m p^{R}$ & Smal & Smal & \\
\hline Fragment 9 & 20110 & 23286 & 3177 & pUC57mini & $A m p^{R}$ & Smal & Smal & \\
\hline Fragment 10 & 22975 & 25940 & 2966 & pUC57mini & $A m p R$ & Smal & Smal & \\
\hline Fragment 11 & 25595 & 28779 & 3185 & pUC57mini & $A m p R$ & Smal & Smal & \\
\hline Fragment 12 & 28274 & 29870 & 1812 & pUC57 & $A m p R$ & Smal & Smal & \\
\hline \multicolumn{9}{|c|}{ pCC1-MERS-CoV-Riyadh-1734-2015 } \\
\hline Fragment 1 & 1 & 2190 & 2339 & & & & & \\
\hline Fragment 8 & 28447 & 30126 & 1791 & pUC57 & $\mathrm{Amp}^{\mathrm{R}}$ & & & \\
\hline \multicolumn{9}{|l|}{ ZIKA virus } \\
\hline Fragment 6 & 10627 & 10807 & 298 & pUC57 & $\mathrm{Amp}^{\mathrm{R}}$ & & & \\
\hline
\end{tabular}


Extended Data Table 4 | Mutations in rSARS-CoV-2(-GFP) YAC clones after sequence confirmation by Sanger sequencing

\begin{tabular}{|c|c|c|c|c|c|c|c|c|c|c|c|c|c|c|c|c|c|c|c|c|c|}
\hline \multirow[b]{2}{*}{ No } & \multicolumn{2}{|c|}{ Mutation location } & \multicolumn{5}{|c|}{ Mutation } & \multicolumn{13}{|c|}{ Clones } & \multirow{2}{*}{ 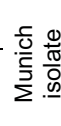 } \\
\hline & $\begin{array}{l}\text { Genome } \\
\text { position }\end{array}$ & $\begin{array}{l}\text { Synthetic } \\
\text { fragment }\end{array}$ & REF & ALT & Type & $\begin{array}{l}\text { Codon } \\
\text { change }\end{array}$ & $\begin{array}{l}\text { Subclones: } \\
\text { AA change }\end{array}$ & 1.1 & 1.2 & 2.1 & 2.2 & 3.1 & 3.2 & 4.1 & 4.2 & 5.1 & 5.2 & 6.1 & 6.2 & ஸे & \\
\hline & 8987 & & $\mathrm{~T}$ & $\mathrm{C}$ & SNP & TTT->CTT & Phe->Leu & $\mathrm{T}$ & C & $\mathrm{T}$ & $\mathrm{T}$ & $\mathrm{T}$ & $\mathrm{T}$ & $\mathrm{T}$ & $\mathrm{T}$ & $\mathrm{T}$ & $\mathrm{T}$ & $\mathrm{T}$ & $\mathrm{T}$ & $\mathrm{T}$ & $\mathrm{T}$ \\
\hline 2 & 9143 & & $\mathrm{~T}$ & $\mathrm{C}$ & SNP & TCT->CCT & Ser->Pro & $\mathrm{T}$ & $\mathrm{T}$ & $\mathrm{T}$ & $\mathrm{T}$ & $\mathrm{T}$ & $\mathrm{T}$ & $\mathrm{T}$ & $\mathrm{T}$ & $\mathrm{T}$ & $\mathrm{T}$ & $\mathrm{C}$ & $\mathrm{T}$ & $\mathrm{T}$ & $\mathrm{T}$ \\
\hline 4 & 11337 & & $\mathrm{~T}$ & C & SNP & TTA->TCA & Leu->Ser & $\mathrm{T}$ & $\mathrm{T}$ & $\mathrm{T}$ & $\mathrm{T}$ & $\mathrm{T}$ & $\mathrm{T}$ & $\mathrm{T}$ & $\mathrm{T}$ & $\mathrm{T}$ & $\mathrm{T}$ & C & $\mathrm{T}$ & $\mathrm{T}$ & $\mathrm{T}$ \\
\hline 5 & 11571 & & $\mathrm{~T}$ & $\mathrm{C}$ & SNP & TTC->TCC & Phe->Ser & $\mathrm{T}$ & $\mathrm{T}$ & $\mathrm{T}$ & $\mathrm{T}$ & $\mathrm{T}$ & $\mathrm{T}$ & $\mathrm{T}$ & $\mathrm{C}$ & $\mathrm{T}$ & $\mathrm{T}$ & $\mathrm{T}$ & $\mathrm{T}$ & $\mathrm{T}$ & $\mathrm{T}$ \\
\hline 6 & 11811 & & G & $A$ & SNP & GGC->GAC & Gly->Asp & G & G & G & G & G & G & G & A & G & G & G & G & G & G \\
\hline 7 & 14580 & & G & $A$ & SNP & ATG->ATA & Met->lle & $\mathrm{G}$ & G & G & G & G & G & $G$ & $G$ & $G$ & $G$ & A & G & $G$ & $G$ \\
\hline 9 & 15531 & & $\mathrm{~T}$ & $\mathrm{C}$ & SNP & TGT->TGC & none (Cys) & $\mathrm{T}$ & $\mathrm{T}$ & $\mathrm{T}$ & $\mathrm{T}$ & $\mathrm{T}$ & $\mathrm{T}$ & $\mathrm{T}$ & $\mathrm{T}$ & $\mathrm{T}$ & $\mathrm{T}$ & C & $\mathrm{T}$ & $\mathrm{T}$ & $\mathrm{T}$ \\
\hline 10 & 15819 & Fragment 7 & $\mathrm{~T}$ & $\varnothing$ & Del & TTA->TAT & Leu->Tyr (frameshift) & $\mathrm{T}$ & $\mathrm{T}$ & $\mathrm{T}$ & $\mathrm{T}$ & $\mathrm{T}$ & $\mathrm{T}$ & $\mathrm{T}$ & $\mathrm{T}$ & $\mathrm{T}$ & $\mathrm{T}$ & $\varnothing$ & $\mathrm{T}$ & $\mathrm{T}$ & $\mathrm{T}$ \\
\hline 11 & 17197 & & G & A & SNP & GCA $->A C A$ & Ala->Thr & G & $A$ & G & G & G & G & G & G & G & G & G & G & G & G \\
\hline 12 & 17432 & & $\mathrm{~T}$ & G & SNP & ATT->AGT & Lys->Ser & $\mathrm{T}$ & $\mathrm{T}$ & $\mathrm{T}$ & $\mathrm{T}$ & $\mathrm{T}$ & G & $\mathrm{T}$ & $\mathrm{T}$ & $\mathrm{T}$ & $\mathrm{T}$ & $\mathrm{T}$ & $\mathrm{T}$ & $\mathrm{T}$ & $\mathrm{T}$ \\
\hline 13 & 17539 & & $G$ & $A$ & SNP & GAC->AAC & Asp->Asn & $G$ & G & G & $\mathrm{G}$ & G & $\mathrm{G}$ & G & $G$ & G & G & $\mathrm{G}$ & $A$ & G & G \\
\hline
\end{tabular}

Genome position indicates the position in the rSARS-CoV-2 genome (MN996528.1). REF indicates the sequence in the rSARS-CoV-2 genome; ALT indicates variation in the rSARS-CoV-2 YAC clone. Codon change shows the effect of the mutation on the corresponding codon. AA change lists the effect of the mutation on the corresponding amino acid. Del, deletion; SNP, single-nucleotide polymorphism; Syn, synSARS-CoV-2-GFP. 


\section{natureresearch}

Corresponding author(s): Volker Thiel

Last updated by author(s): 2020/04/15

\section{Reporting Summary}

Nature Research wishes to improve the reproducibility of the work that we publish. This form provides structure for consistency and transparency in reporting. For further information on Nature Research policies, see Authors \& Referees and the Editorial Policy Checklist.

\section{Statistics}

For all statistical analyses, confirm that the following items are present in the figure legend, table legend, main text, or Methods section.

n/a Confirmed

$\square \bigotimes$ The exact sample size $(n)$ for each experimental group/condition, given as a discrete number and unit of measurement

$\square$ \A statement on whether measurements were taken from distinct samples or whether the same sample was measured repeatedly

$\triangle$ The statistical test(s) used AND whether they are one- or two-sided

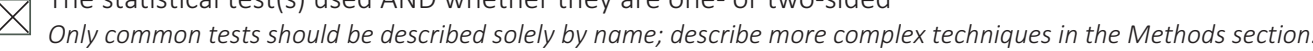

$\bigotimes \square$ A description of all covariates tested

$\square$ A description of any assumptions or corrections, such as tests of normality and adjustment for multiple comparisons

$\triangle \square$ A full description of the statistical parameters including central tendency (e.g. means) or other basic estimates (e.g. regression coefficient) $\triangle$ AND variation (e.g. standard deviation) or associated estimates of uncertainty (e.g. confidence intervals)

$\bigotimes$ For null hypothesis testing, the test statistic (e.g. $F, t, r$ ) with confidence intervals, effect sizes, degrees of freedom and $P$ value noted

$\square$ Give $P$ values as exact values whenever suitable.

Х $\square$ For Bayesian analysis, information on the choice of priors and Markov chain Monte Carlo settings

Х $\square$ For hierarchical and complex designs, identification of the appropriate level for tests and full reporting of outcomes

Х $\square$ Estimates of effect sizes (e.g. Cohen's $d$, Pearson's $r$ ), indicating how they were calculated

Our web collection on statistics for biologists contains articles on many of the points above.

\section{Software and code}

Policy information about availability of computer code

Data collection Data acquisition for Minion sequencing was done with MinKNOW version 19.06 .9 and basecalling software Guppy version 3.4.5.

Data analysis $\quad$ seqeunce analysis: Geneious Prime ${ }^{\circledR}$ 2019.2.3

virus kinetics analyses: GraphPad Prism version 8.3.0 for Windows

figures: Adobe Illustrator and Biorender

IFA: FIJI with FigureJ plugin

NGS (RNAseq): TrimGalore software (version 0.6.5), STAR (version 2.7.0a); SAMtools (version 1.10);

Minion sequencing: Python command-line qcat (Mozilla Public License 2.0. Copyright (C 2018 Oxford Nanopore Technologies Ltd. qcat

(v1.1.0); Minimap2 (Li, H. Minimap2: pairwise alignment for nucleotide sequences. Bioinformatics 34, 3094-3100, doi:10.1093/

bioinformatics/bty191 (2018).)

For manuscripts utilizing custom algorithms or software that are central to the research but not yet described in published literature, software must be made available to editors/reviewers. We strongly encourage code deposition in a community repository (e.g. GitHub). See the Nature Research guidelines for submitting code \& software for further information.

Data

Policy information about availability of data

All manuscripts must include a data availability statement. This statement should provide the following information, where applicable:

- Accession codes, unique identifiers, or web links for publicly available datasets

- A list of figures that have associated raw data

- A description of any restrictions on data availability

All Data and files will be made available. The following genome sequences have been submitted to GenBank: rSARS-CoV-2 (\#MT108784), hRSV/B/Bern/2019 (\#MT107528); MERS-CoV-Riyadh-1734-2015 (\#MN481979). The RNAseq data of rSARS-CoV-2(-GFP) has been submitted to the NCBI Sequence Read Archive 


\section{Field-specific reporting}

Please select the one below that is the best fit for your research. If you are not sure, read the appropriate sections before making your selection. $\bigotimes$ Life sciences $\quad \square$ Behavioural \& social sciences $\quad \square$ Ecological, evolutionary \& environmental sciences

\section{Life sciences study design}

All studies must disclose on these points even when the disclosure is negative.

Sample size No sample size calculations were performed. Sample sizes were based on standards in the field, typically 3 independent biological replicates, with each replicate assayed in technical duplicate or triplicate.

Data exclusions no data was excluded

Replication all attempts at replication were successful; experiments were performed according to best practices and as described in the methods.

Randomization randomization was not applied since cloning procedures, virus infection/titrations, and inhibitor/neutralization experiments did not require randomization.

Blinding blinding was done for remdsivir inhibition assay and virus neutralisation assay to ensure that images taken from infected cultures are representative.

\section{Reporting for specific materials, systems and methods}

We require information from authors about some types of materials, experimental systems and methods used in many studies. Here, indicate whether each material, system or method listed is relevant to your study. If you are not sure if a list item applies to your research, read the appropriate section before selecting a response.

Materials \& experimental systems

$\mathrm{n} / \mathrm{a}$ Involved in the study

$\square \bigotimes$ Antibodies

$\square \bigotimes$ Eukaryotic cell lines

$\bigotimes \square$ Palaeontology

$\bigotimes \square$ Animals and other organisms

$\bigotimes \square$ Human research participants

$\bigotimes \square$ Clinical data

\begin{tabular}{l|l}
\multicolumn{2}{l}{ Methods } \\
\hline n/a & Involved in the study \\
$X$ & $\square$ ChIP-seq \\
$X$ & $\square$ Flow cytometry \\
$\square$ & $\square$ MRI-based neuroimaging
\end{tabular}

\section{Antibodies}

Antibodies used

anti-SARS-CoV Nucleocapsid (N) protein (rabbit), Rockland, Product No: 200-401-50, Lot No 16570, dillution 1:1000; anti-dsRNA, J2, English and Scientific Consulting, Product No:10010500, clone J2, Lot No J2-1913, dillution 1:200.

Validation

anti-SARS-CoV Nucleocapsid (N) protein (rabbit) and anti-dsRNA, J2 were validated by comparing infected vs uninfected cells and by assessing various dillutions.

\section{Eukaryotic cell lines}

Policy information about cell lines

Cell line source(s)

L929 cells (Source : 85011425 (Sigma, ECACC, 2017); authenticated 04/2019);

17Cl-1 cells (gift from Stanley Sawicki; authenticated 04/2019);

Huh7 cells (gift from Volker Lohman, University of Heidelberg; authetication was done in Heidelberg)

Vero, VeroB4 and VeroE6 (obtained from Marcel Müller, Charité, Berlin (co-author); authentication done in Berlin

Authentication

Profiling of cell line was done using highly-polymorphic short tandem repeat loci (STRs). Fragment analysis was done on an ABI3730xI (Life Technologies) and the resulting data were analyzed with GeneMarker software (Softgenetics). 
